Review

\title{
Polymeric Drug Delivery Systems Bearing Cholesterol Moieties: A Review
}

\author{
Paweł Misiak *(D), Karolina H. Markiewicz, Dawid Szymczuk(D) and Agnieszka Z. Wilczewska * \\ Faculty of Chemistry, University of Bialystok, Ciolkowskiego 1k, 15-245 Bialystok, Poland; \\ k.markiewicz@uwb.edu.pl (K.H.M.); d.szymczuk@uwb.edu.pl (D.S.) \\ * Correspondence: p.misiak@uwb.edu.pl (P.M.); agawilcz@uwb.edu.pl (A.Z.W.); Tel.: +48-85-7388037
}

Received: 30 September 2020; Accepted: 4 November 2020; Published: 6 November 2020

\begin{abstract}
This review aims to provide an overview of polymers comprising cholesterol moiety/ies designed to be used in drug delivery. Over the last two decades, there have been many papers published in this field, which are summarized in this review. The primary focus of this article is on the methods of synthesis of polymers bearing cholesterol in the main chain or as side chains. The data related to the composition, molecular weight, and molecular weight distribution of polymers are presented. Moreover, other aspects, such as forms of carriers, types of encapsulated drugs, encapsulation efficiency and capacity, are also included.
\end{abstract}

Keywords: cholesterol; polymers comprising cholesteryl moiety; drug delivery; drug encapsulation; encapsulation efficiency; encapsulation capacity

\section{Introduction}

The inability to use the potential of available bioactive substances is an important issue of modern medicine. Many of the known drug molecules are successful when colliding with cells of bacteria, fungi, and tumors. However, their application in a conventional form is characterized by limited effectiveness due to low solubility, poor biodistribution, poor stability and rapid clearance from the body [1,2]. Therefore, smart drug delivery systems (DDS) are widely investigated and developed to improve the effectiveness of therapy by optimizing the dose and duration of drug action directly in the target site [1,3-5].

A variety of DDS, such as stimuli-responsive polymeric nanoparticles, liposomes, organic-inorganic hybrids, and exosomes, has been reported in scientific journals [6-10]. Among them, polymers are promising drug carriers because of the flexibility in the control of chemical compositions and functions of macromolecules (drug conjugation, stimulus sensitivity, stealth properties, specific targeting, etc.). A plethora of polymers has been used to obtain carriers with an innumerable variety of physicochemical and biological properties. The most frequently used are biocompatible and biodegradable polymers of natural or synthetic origin such as chitosan (CS) [11-13] hyaluronic acid (HA) [13-17], peptides [18], N-(2-hydroxypropyl)methacrylamide (HPMA) [19-27], poly(ethylene glycol) (PEG) [25,26,28-75], poly(glutamic acid) (PGA) [53,76,77], poly(lactic acid) (PLA) [28,78,79], and poly(D,L-lactide-co-glycolide) (PLGA) $[29,53,80]$. Their advantages are low toxicity, reduction of possible side effects, and ease of excretion $[3,4,81]$.

One of the critical issues related to the efficiency of smart drug nanocarriers is their interaction with cell membranes. The modification of a carrier structure with a cell-penetrating ligand is a strategy to improve cellular uptake [82,83]. Cholesterol (Figure 1) is an organic compound, a steroid lipid, which is an essential structural component of animal cell membranes. It is responsible for the integration, fluidity, microdomain structure (so-called lipid rafts), and the permeability of the membrane. Cholesterol owes these properties to its structure-the hydroxyl group interacts with 
water molecules similar to the hydrophilic main groups of phospholipids, while the carbon skeleton shows a high affinity for the hydrophobic tails of phospholipids. The rigid and flat tetracyclic structure regulates the fluidity of the cell membrane $[84,85]$. Furthermore, cholesterol is a precursor in the biosynthesis of a wide range of biologically important substances, including bile acids, vitamin D, and sex hormones [86,87].

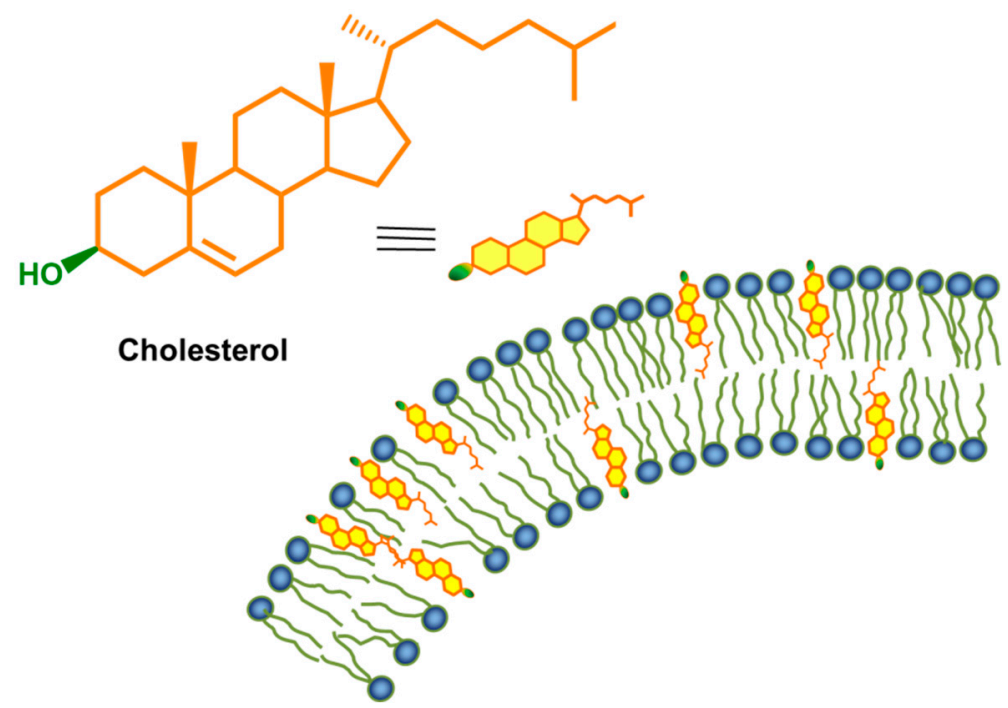

Figure 1. The structural formula of cholesterol and interaction in lipid bilayer.

Due to its hydroxyl group which can easily be derivatized, large-scale availability, and relatively low cost, cholesterol has been used as a starting material for the synthesis of diverse steroid-based molecules [88,89]. High biocompatibility and the ability to be incorporated into cell membranes make cholesterol and its derivatives increasingly used in DDS.

In this review, we present an overview of numerous publications devoted to polymers comprising cholesterol designed for drug delivery. We describe the methods of incorporation of cholesterol moiety/ies into a polymer chain and the forms of drug carriers that have been obtained using these polymers. We present the types of drugs that have been encapsulated and the effectiveness (encapsulation efficiency and capacity) of their loading. Some examples of polymers bearing cholesterol moieties designed for drug delivery have been featured in more general review covering the topic of cholesterol chemistry and its applications in different research fields [88]. Moreover, there has been one earlier review concerning polymers comprising cholesterol, published in 2009 [90]. It was mainly focused on synthesis and strategies of direct ordering and packing of meso- and nanostructures of cholesterol polymers in the neat or melt state and in solution. It also dealt with their various applications, including drug delivery. However, the topic of drug delivery systems based on polymers comprising cholesterol has moved forward significantly since then.

\section{Methods of Synthesis of Polymers Containing Cholesterol}

A variety of polymerization methods and selective chemical reactions allow for the obtention of polymers with cholesterol incorporated both in the main chain and in side chains $[88,90]$. Due to the methodology of their preparation, polymers comprising cholesterol can be divided into polymers obtained (I) by polymerization of cholesterol-containing monomers, (II) by post-modification of side chains, (III) using a chain transfer agent or an initiator containing cholesterol, and (IV) as a result of chain end post-modification (Figure 2). 




Figure 2. Methods of synthesis of polymers containing cholesteryl moieties.

\subsection{Polymers Containing Cholesterol in the Main Chain}

There are two approaches that give the possibility to introduce cholesterol moiety at the end of the polymeric chain: the use of cholesterol-containing initiators or chain transfer agents (Figure 3, Table 1) and post-modification of the reactive chain end (Figure 4, Table 2). These approaches result in polymers with one cholesteryl moiety. In the context of drug delivery, recent studies [91] show that even with a relatively long polymer ballast, it is possible to take advantage of the properties of cholesterol present at the polymer chain end. In such systems, cholesterol plays primarily a guiding role, but it can also integrate into the biological membrane, which may allow releasing drug molecules in the immediate vicinity or even inside a pathological cell. Considering solubility in physiological fluids, one hydrophobic cholesterol moiety is an advantage, as such a system does not have to contain excessively large hydrophilic part to be soluble.

\subsubsection{Cholesterol Introduced to the Main Chain during Polymerization}

Free radical polymerization (FRP) is the simplest method used in the synthesis of DDS. Due to the non-specific nature of free radicals, FRP is a versatile method that allows the polymerization of most vinyl monomers. The advantage of this type of polymerization is that it is not an expensive and fast method. Additionally, isolation and purification of the product are relatively easy. The significant disadvantages of FRP include high dispersity of the obtained systems and dead-end product, due to the termination processes, which preclude the copolymerization of subsequent blocks. The twentieth century was rich in the development of new polymerization methods, atom transfer radical polymerization (ATRP) in 1995 [92,93], and reversible addition-fragmentation chain transfer polymerization (RAFT) in 1998 [94]. With the growing interest in the field of drug delivery, polymeric carriers, star systems, dendrimers, and nanogels began to be used. Such structures are easier to obtain using CRP methods as compared to conventional FRP. Another advantage of controlled radical polymerization is the eventuality of obtaining a system with lower dispersity and the possibility of creating copolymer libraries that originate from one precursor polymer. For instance, in RAFT polymerization, there is 
a RAFT agent at the end of the chain, which can be re-initiated to propagate other blocks [95]. Additionally, functionalized monomers can be polymerized by CRP techniques. In the group of radical polymerizations with reversible deactivation (RDRP), the RAFT method has the greatest tolerance on reactive functional groups [95]. On the other hand, there is a multitude of types of ATRP, resulting in the high flexibility of this method [96]. CRP methods are not perfect and free from drawbacks, particularly when making use of the drug delivery systems thus obtained. Often, it is not possible to avoid the use of toxic initiators, which contain transition metals such as iron, copper, tin, or osmium (ATRP) [97]. In RAFT polymerization, it is necessary to use an additional factor which is the chain transfer agent (CTA), also called the RAFT agent. Dithiocarbonates and trithiocarbonates, which are used the most often, remain at the end of the polymer chain. This can also be a big disadvantage because these ends show toxic properties for the human body. However, at the same time, they allow for further copolymerization or appropriate modification, e.g., to the thiol group [98], which opens up a variety of possibilities from the Michael reaction to the formation of disulfides.

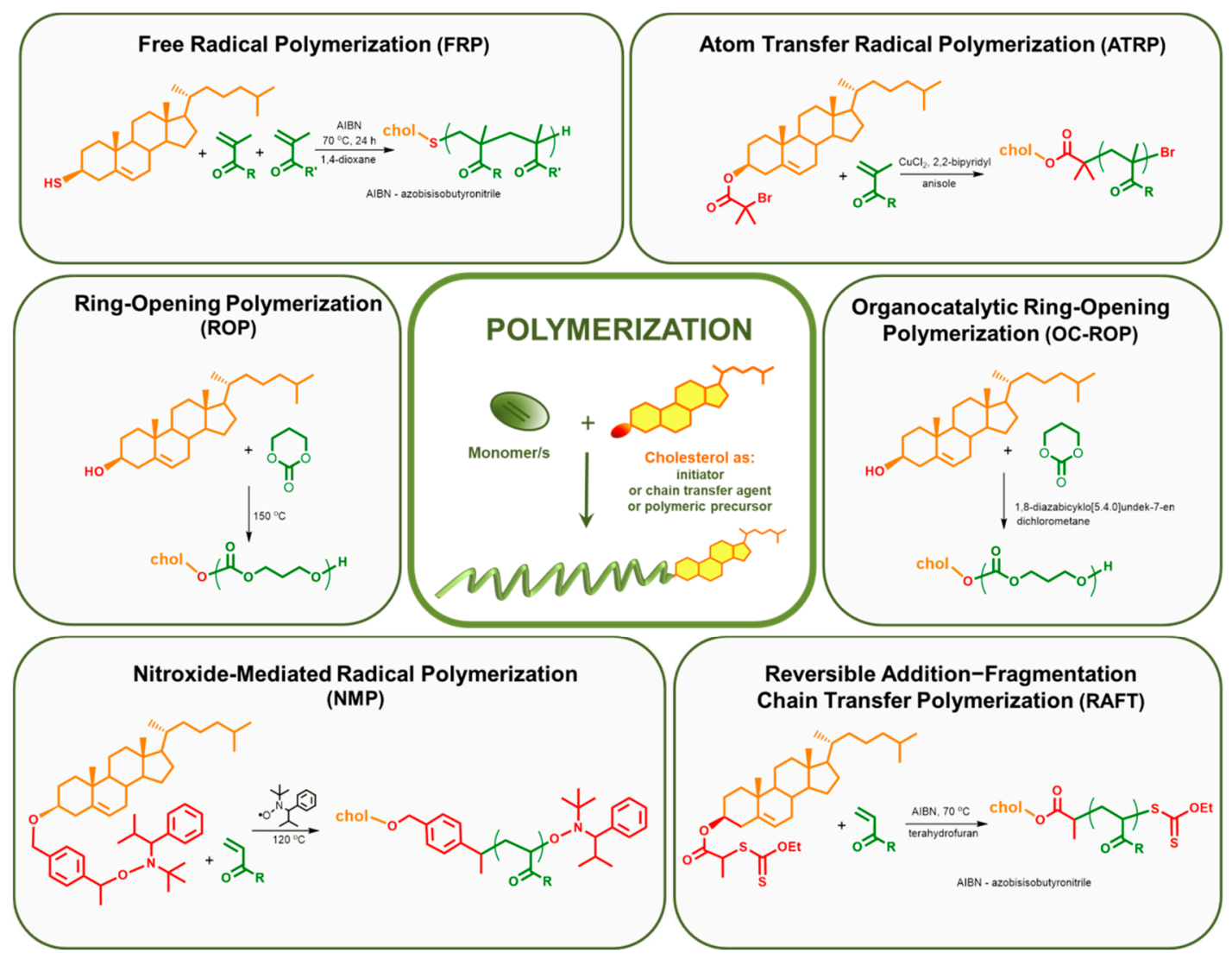

Figure 3. Polymerization methods used for the synthesis of cholesterol end-capped polymers and reaction examples $[24,52,54,91,99-101]$. 
Table 1. Cholesterol end-capped polymers reported as drug delivery systems.

\begin{tabular}{|c|c|c|c|c|c|c|}
\hline Polymer & Form of Carrier & Drug or Dye & $M_{n}$ or $M_{w}(k D a)(Đ)$ & LE (\%) & LC (\%) & Lit. \\
\hline \multicolumn{7}{|c|}{ Free Radical Polymerization (FRP) } \\
\hline Chol-pHPMAlac (mono:di $=30: 70$ ) & \multirow{4}{*}{ liposome } & \multirow{4}{*}{ DOX } & $10.5(1.60-1.70)$ & 93.0 & \multirow{4}{*}{ N/A } & \multirow{4}{*}{ [24] } \\
\hline Chol-pHPMAlac (mono:di $=44: 56$ ) & & & $10.0(1.60-1.70)$ & 99.0 & & \\
\hline Chol-pHPMAlac (mono:di $=54: 46$ ) & & & $11.0(1.60-1.70)$ & 100 & & \\
\hline Chol-pHPMAlac (mono:di $=67: 33$ ) & & & $11.0(1.60-1.70)$ & 100 & & \\
\hline \multicolumn{7}{|c|}{ Nitroxide-Mediated Controlled Radical Polymerization (NMP) } \\
\hline Chol-PAA & PCLp & siRNA & N/A & 46.0 & 0.8 & {$[100,101]$} \\
\hline \multicolumn{7}{|c|}{ Atom Transfer Radical Polymerization (ATRP) } \\
\hline \multirow{2}{*}{ Chol-PDMAEMA } & \multirow{2}{*}{ liposome } & $\mathrm{CF}$ & \multirow{2}{*}{$5.4(1.17)$} & \multirow{2}{*}{$\mathrm{N} / \mathrm{A}$} & \multirow{2}{*}{$\mathrm{N} / \mathrm{A}$} & \multirow{2}{*}{ [102] } \\
\hline & & calcein & & & & \\
\hline Chol-PAA $5 \%$ in lip & \multirow{4}{*}{ liposome } & \multirow{4}{*}{ calcein } & \multirow{4}{*}{$7.2(\mathrm{~N} / \mathrm{A})$} & 29.6 & \multirow{4}{*}{ N/A } & \multirow{4}{*}[103,104]{} \\
\hline Chol-PAA $10 \%$ in lip & & & & 46.1 & & \\
\hline Chol-PAA 20\% in lip & & & & 28.8 & & \\
\hline Chol-PAA $10 \%$ in lip crosslinked & & & & 24.7 & & \\
\hline Chol-LC-PDMAEMA & liposome & calcein & N/A & $\mathrm{N} / \mathrm{A}$ & $\mathrm{N} / \mathrm{A}$ & [105] \\
\hline Chol-PLA-SS-PMPC & micelle & Nile red & N/A & N/A & N/A & [79] \\
\hline \multirow{6}{*}{ Chol-b-pMPC } & \multirow{3}{*}{ polymersome } & \multirow{3}{*}{ ADR } & $6.4(\mathrm{~N} / \mathrm{A})$ & 3.6 & \multirow{3}{*}{ N/A } & \multirow{3}{*}{ [106] } \\
\hline & & & 9.5 (N/A) & 4.2 & & \\
\hline & & & 15.4 (N/A) & 4.0 & & \\
\hline & \multirow{3}{*}{ micelle } & \multirow{3}{*}{$\mathrm{ADR}$} & 3.0 (N/A) & \multirow{3}{*}{$\mathrm{N} / \mathrm{A}$} & \multirow{3}{*}{ N/A } & \multirow{3}{*}{ [107] } \\
\hline & & & $6.4(\mathrm{~N} / \mathrm{A})$ & & & \\
\hline & & & N/A & & & \\
\hline \multirow{3}{*}{ Chol-PEO } & \multirow{3}{*}{ micelle } & \multirow{3}{*}{ ADR } & $1.7(1.13)$ & & 10.1 & \\
\hline & & & $2.3(1.10)$ & $\mathrm{N} / \mathrm{A}$ & 16.2 & [108] \\
\hline & & & $2.8(1.10)$ & & 16.9 & \\
\hline Chol-PEGMA $_{50}$ & & & $33.2(1.25)$ & & 15.6 & \\
\hline Chol-PEGMA $_{100}$ & micelle & $\mathrm{QC}$ & $52.2(1.32)$ & $\mathrm{N} / \mathrm{A}$ & 14.1 & [54] \\
\hline Chol-PEGMA $_{200}$ & & & $89.1(1.55)$ & & 14.1 & \\
\hline
\end{tabular}


Table 1. Cont.

\begin{tabular}{|c|c|c|c|c|c|c|}
\hline Polymer & Form of Carrier & Drug or Dye & $M_{n}$ or $M_{w}(k D a)(Đ)$ & LE $(\%)$ & LC (\%) & Lit. \\
\hline \multicolumn{7}{|c|}{ Reversible Addition-Fragmentation Chain Transfer Polymerization (RAFT) } \\
\hline \multirow{5}{*}{ Chol-PNIPAAm } & \multirow{5}{*}{ micelle } & \multirow{5}{*}{ N/A } & $3.2(1.27)$ & \multirow{5}{*}{$\mathrm{N} / \mathrm{A}$} & \multirow{5}{*}{ N/A } & \multirow{5}{*}{ [91] } \\
\hline & & & $5.7(1.35)$ & & & \\
\hline & & & $6.1(1.51)$ & & & \\
\hline & & & $8.4(1.64)$ & & & \\
\hline & & & $10.9(1.90)$ & & & \\
\hline
\end{tabular}

Ring-Opening Polymerization (ROP)

\begin{tabular}{|c|c|c|c|c|c|c|}
\hline & \multirow{5}{*}{ nanoparticle } & \multirow{5}{*}{ prednisone acetate } & & \multirow{5}{*}{ N/A } & \multirow{5}{*}{ N/A } & \multirow{5}{*}{ [109] } \\
\hline Chol-PCL (nChol:nPCL = 1:10) & & & $5.5(1.34)$ & & & \\
\hline Chol-PCL (nChol:nPCL = 1:20) & & & $7.2(1.55)$ & & & \\
\hline Chol-PCL (nChol:nPCL = 1:40) & & & $11.4(1.69)$ & & & \\
\hline Chol-PCL (nChol:nPCL = 1:80) & & & $16.2(1.79)$ & & & \\
\hline Chol-pTMC (nChol:nTMC = 1:4) & \multirow{5}{*}{ nanoparticle } & \multirow{5}{*}{ prednisone acetate } & $1.8(1.26)$ & & & \multirow{5}{*}{ [99] } \\
\hline Chol-pTMC (nChol:nTMC = 1:10) & & & $2.7(1.75)$ & N/A & N/A & \\
\hline Chol-pTMC (nChol:nTMC = 1:20) & & & $5.2(1.78)$ & & & \\
\hline Chol-pTMC (nChol:nTMC = 1:40) & & & $9.7(1.65)$ & 61.7 & 9.1 & \\
\hline Chol-pTMC (nChol:nTMC = 1:80) & & & $13.9(1.80)$ & N/A & N/A & \\
\hline \multicolumn{7}{|c|}{ Organocatalytic Ring-Opening Polymerization (OC-ROP) } \\
\hline Chol-PTMC-PEG & nanoparticle & DOX & $6.6(\mathrm{~N} / \mathrm{A})$ & N/A & 7.3 & [52] \\
\hline Chol-PTMC & \multirow{4}{*}{ surface } & \multirow{4}{*}{ FITC-BSA } & $11.3(1.20)$ & \multirow{4}{*}{ N/A } & \multirow{4}{*}{ N/A } & \multirow{4}{*}{ [78] } \\
\hline Chol-PTMC-PLA & & & $10.1(1.40)$ & & & \\
\hline Chol-PTMC-PMBC & & & $2.5(1.20)$ & & & \\
\hline Chol-PMBC & & & $3.3(1.50)$ & & & \\
\hline
\end{tabular}

Abbreviations: ADR, Adriamycin; ATRP, atom transfer radical polymerization; Chol, cholesterol; CF, 5,6-carboxyfluorescein; DMAEMA, 2-(dimethylamino)ethyl methacrylate; DOX, doxorubicin; FITC-BSA, fluorescein isothiocyanate-labeled bovine serum albumin; LC, lecithin; MBC, 5-methyl-5-benzylcarboxyl-1,3-dioxan-2-one; MPC, 2-methacryloyloxyethyl phosphorylcholine; NIPAAm, $N$-isopropylacrylamide; OC-ROP, organocatalytic ring-opening polymerization; PAA, poly(acrylic acid); PCL, poly( $\varepsilon$-caprolactone); PCLp, polymer-caged lipoplex; PEG, poly(ethylene glycol); PEGMA, poly(ethylene glycol) methyl ether methacrylate; PEO, poly(ethylene oxide); PHPMAlac, poly(N-(2-hydroxypropyl)methacrylamide mono/dilactate); PLA, poly(lactic acid); RAFT, reversible addition-fragmentation chain transfer polymerization; ROP, ring-opening polymerization; TMC, trimethylene carbonate; $\mathrm{QC}$, quercetin. 
The majority of the methods used in the synthesis of polymeric drug delivery systems with an incorporated cholesterol molecule are controlled radical polymerizations such as ATRP [54,79,102-108], RAFT [91] or NMP [100] (Table 1). This is due to the possibility of controlling the dispersion of the system, which translates into stability in biological properties and accuracy in predicting the behavior of the carrier in the human body. Ring-opening polymerization techniques are also widely used, and most popular is the polymerization of cyclic monomers such as $\mathcal{\varepsilon}$-caprolactone [109] and trimethylene carbonate $[51,78,99]$.

\subsubsection{Cholesterol Introduced to the Main Chain by Post-Modification}

The chemistry of polymers makes it possible to obtain functional macromolecules in a simple, fast, and relatively inexpensive way. However, in some cases, the presence of certain functional groups makes it impossible to perform polymerization. Post-polymerization modification, which is a combination of the achievements of polymer chemistry and organic synthesis, comes to the rescue. It consists of the preparation of a polymer that has modifiable, available groups, which are then subjected to various reactions from simple esterification ( $\mathrm{O}$-acylation) or amidation ( $\mathrm{N}$-acylation) through various coupling reactions to click reactions (Figure 4). The advantage of this methodology is the formation of functional products that are impossible to obtain by polymerization. The post-modification approach allows the creation of a library of functional polymers based on one reactive precursor, which ensures the maintenance of the same structural parameters such as tacticity, molecular weight distribution, or the degree of polymerization. In many cases, it turns out that the polymerization of a commercially available monomer and the subsequent functionalization of the polymer is a less time-consuming and cost-intensive method than the synthesis and polymerization of an original monomer. Additionally, the storage of the reactive monomer is at greater risk than the polymeric precursor [110,111]. On the other hand, it should be remembered that the post-polymerization approach has some important limitations. In the case of the functionalization of polymer precursors, it should be taken into account that organic reactions do not run with a $100 \%$ yield. This is caused by many factors, such as (I) availability of reactive groups; (II) possible steric hindrance in the polymer chain; (III) curling, twisting the polymer; or (IV) the need for additional purification, either from catalysts or other reactants used.

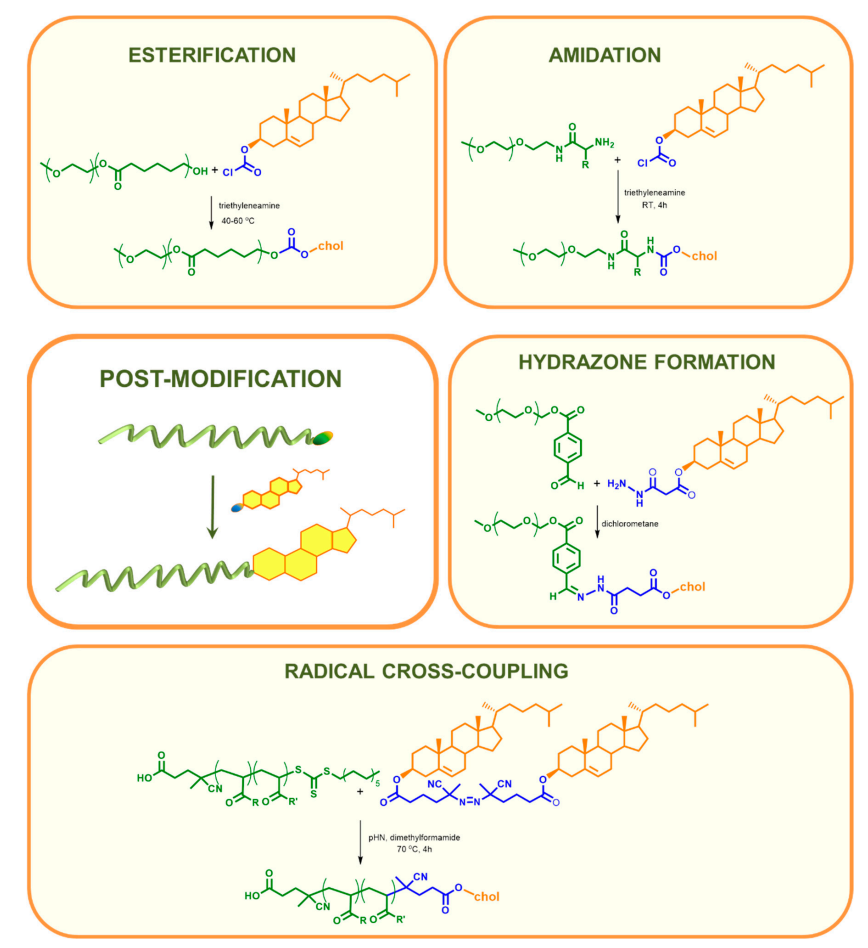

Figure 4. Post-modification reactions used to introduce cholesteryl moieties at the end of the polymeric chain $[27,58,69,74]$. 
Table 2. Cholesterol end-capped polymers obtained by post-modification reported as DDS.

\begin{tabular}{|c|c|c|c|c|c|c|}
\hline Polymer & Form of Carrier & Drug or Dye & $\mathrm{M}_{\mathrm{n}}$ or $\mathrm{M}_{\mathrm{w}}(\mathrm{kDa})(\bigoplus)$ & LE (\%) & LC (\%) & Lit. \\
\hline \multicolumn{7}{|c|}{ Esterification } \\
\hline \multirow{3}{*}{ Acetylene- $\mathrm{PEG}_{10 \mathrm{~K}}-\mathrm{G}_{4}-\mathrm{Chol}_{16}$} & \multirow{4}{*}{ micelle } & DOX & \multirow{3}{*}{$14.2(1.11)$} & \multirow{3}{*}{ N/A } & 18.8 & \multirow{4}{*}{ [55] } \\
\hline & & TPL & & & 8.4 & \\
\hline & & $\mathrm{DOX}+\mathrm{TPL}$ & & & N/A/6.5 & \\
\hline Rhodamine-PEG ${ }_{10 \mathrm{~K}}-\mathrm{G}_{4}-\mathrm{Chol}_{16}$ & & DOX & $17.6(1.14)$ & N/A & 10.0 & \\
\hline mPEG-Chol & micelle & DTXL & N/A & 97.6 & 4.8 & [56] \\
\hline \multirow{4}{*}{ mPEG-Chol/RGD-mPEG-Chol (10\% mPEG-Chol $w / w)$} & \multirow{8}{*}{ liposome } & $\operatorname{PTX}(2.5 \% w / w)$ & \multirow{8}{*}{ N/A } & 99.8 & 0.05 & \multirow{8}{*}{ [57] } \\
\hline & & $\operatorname{PTX}(5 \% w / w)$ & & 99.6 & 0.08 & \\
\hline & & $\operatorname{PTX}(7.5 \% w / w)$ & & 99.1 & 1.15 & \\
\hline & & $\operatorname{PTX}(10 \% w / w)$ & & 97.3 & 1.62 & \\
\hline \multirow{4}{*}{ mPEG-Chol/RGD-mPEG-Chol (20\% mPEG-Chol $w / w)$} & & $\operatorname{PTX}(2.5 \% w / w)$ & & 99.7 & 0.53 & \\
\hline & & $\operatorname{PTX}(5 \% w / w)$ & & 99.1 & 0.81 & \\
\hline & & $\operatorname{PTX}(7.5 \% w / w)$ & & 98.9 & 1.06 & \\
\hline & & $\operatorname{PTX}(10 \% w / w)$ & & 95.1 & 1.48 & \\
\hline mPEG-Chol & \multirow{2}{*}{ micelle } & \multirow{2}{*}{$\mathrm{AmB}$} & $5.9(1.04)$ & 42.0 & 8.8 & \multirow{2}{*}{ [58] } \\
\hline mPEG- $b$-PCL-Chol & & & $10.1(1.20)$ & 60.0 & 12.5 & \\
\hline TPGS-Chol & micelle & DTXL & N/A & 99.2 & 3.2 & [112] \\
\hline Chol-PEG-GA & liposome & brucine & N/A & 82.5 & N/A & [59] \\
\hline Chol-PEG ${ }_{2 K} /(\gamma-$ PGA- $g$-PLGA $)$ & \multirow{6}{*}{ nanoparticle } & & \multirow{6}{*}{ N/A } & 63.8 & 4.6 & \multirow{6}{*}{ [53] } \\
\hline Chol-PEG $_{5 \mathrm{~K}} /(\gamma$-PGA- $g$-PLGA $)$ & & DOX & & 66.8 & 4.8 & \\
\hline Chol-PEG ${ }_{10 K} /(\gamma$-PGA- $g$-PLGA $)$ & & & & 66.6 & 4.7 & \\
\hline Chol-PEG $2 \mathrm{~K} /(\gamma$-PGA-g-PLGA $)$ & & \multirow{3}{*}{ ICG } & & 86.8 & 6.2 & \\
\hline Chol-PEG $_{5 \mathrm{~K}} /(\gamma$-PGA- $g$-PLGA $)$ & & & & 86.8 & 6.2 & \\
\hline Chol-PEG $_{10 K} /(\gamma$-PGA- $g$-PLGA $)$ & & & & 84.9 & 6.1 & \\
\hline PF127-Chol & \multirow{2}{*}{ micelle } & \multirow{2}{*}{$\begin{array}{l}\text { DTXL (temp., ratio, } \\
\text { solvent) }\end{array}$} & \multirow{2}{*}{ N/A } & 81.0 & \multirow{2}{*}{ N/A } & \multirow{2}{*}[113]{} \\
\hline FA-PF127-Chol & & & & $65.4-103.2$ & & \\
\hline
\end{tabular}


Table 2. Cont

\begin{tabular}{|c|c|c|c|c|c|c|}
\hline Polymer & Form of Carrier & Drug or Dye & $M_{n}$ or $M_{w}(k D a)(Đ)$ & LE (\%) & LC (\%) & Lit. \\
\hline Chol-PSO & \multirow{2}{*}{ micelle } & \multirow{2}{*}{ PTX } & 2.4 & 80.1 & 18.6 & \multirow{2}{*}{ [114] } \\
\hline Chol-PSO-(HE) $)_{5}$-Fmoc/Chol-PSO-(RG) $)_{5}$-Pbf & & & N/A & 78.5 & 17.1 & \\
\hline F68-Chol & micelle & CABA & N/A & 98.1 & 3.2 & [115] \\
\hline mPEG-Chol & micelle & QC & N/A & 93.5 & 3.7 & {$[60]$} \\
\hline Biotin-PAE- $g$-mPEG-Chol & \multirow{2}{*}{ micelle } & \multirow{2}{*}{ DOX } & $11.8(1.60)$ & 61.0 & 5.5 & \multirow{2}{*}{ [61] } \\
\hline PAE-g-mPEG-Chol & & & N/A & 47.0 & 4.2 & \\
\hline mPEG-PLA-Chol & micelle & CUR & N/A & 93.7 & 11.9 & [28] \\
\hline PEG-PLLA-Chol & \multirow{2}{*}{ micelle } & \multirow{2}{*}{ DOX } & \multirow{2}{*}{ N/A } & 45.3 & 8.3 & \multirow{2}{*}[62,63]{} \\
\hline PEG-PDLA-Chol & & & & 48.2 & 8.8 & \\
\hline Chol-PEG & micelle & PTX & N/A & $>90$ & N/A & [64] \\
\hline Chol-PEG-DUP1 & micelle & PTX & $\mathrm{N} / \mathrm{A}$ & 96.4 & 24.9 & [65] \\
\hline \multirow{5}{*}{ Chol-mPEG-RGD/mPEG-PLGA } & \multirow{5}{*}{ nanoparticle } & CUR $(2 \% w / w)$ & \multirow{5}{*}{$\mathrm{N} / \mathrm{A}$} & 100 & 2.00 & \multirow{5}{*}{ [29] } \\
\hline & & CUR $(3 \% w / w)$ & & 98.7 & 2.96 & \\
\hline & & CUR $(4 \% w / w)$ & & 97.8 & 3.91 & \\
\hline & & CUR $(5 \% w / w)$ & & 96.0 & 4.80 & \\
\hline & & CUR $(7 \% w / w)$ & & 70.7 & 4.95 & \\
\hline P(NIPAAm-co-DMAAm)-g-Chol & \multirow{2}{*}{ micelle } & \multirow{2}{*}{ Py } & $2.9(1.20)$ & \multirow{2}{*}{ N/A } & $0.8 \mathrm{mg} / \mathrm{g}$ & \multirow{2}{*}{ [116] } \\
\hline P(NIPAAm-co-DMAAm)-g-Chol & & & $6.4(1.30)$ & & $1 \mathrm{mg} / \mathrm{g}$ & \\
\hline Chol-PEG-TPP & liposome & $\mathrm{CF}$ & N/A & 1.8 & $\mathrm{~N} / \mathrm{A}$ & [66] \\
\hline mPEG- $b$-PCL-Chol & micelle & CUR & $6.6(1.17)$ & 32.0 & 8.8 & [117] \\
\hline \multicolumn{7}{|c|}{$\begin{array}{ll}\text { Amidation } \\
\end{array}$} \\
\hline Chol-PEG-PpIX & micelle anchored to liposome & itself & N/A & N/A & N/A & [67] \\
\hline HA-SA-CYS-Chol & micelle & DTXL & $30.1(1.70)$ & 89.7 & 4.8 & [16] \\
\hline \multirow{3}{*}{ HA-Chol } & \multirow{3}{*}{ nanoparticle } & DTXL & & 66.9 & 1.9 & \multirow{3}{*}{ [17] } \\
\hline & & TMX & $\mathrm{N} / \mathrm{A}$ & 76.5 & 4.1 & \\
\hline & & DTXL/TMX & & $83.1 / 92.5$ & $1.4 / 3.4$ & \\
\hline
\end{tabular}


Table 2. Cont

\begin{tabular}{|c|c|c|c|c|c|c|}
\hline Polymer & Form of Carrier & Drug or Dye & $\mathrm{M}_{\mathrm{n}}$ or $\mathrm{M}_{\mathrm{w}}(\mathrm{kDa})(\boxplus)$ & LE (\%) & LC (\%) & Lit. \\
\hline PEG-PAsp(DET)-Chol & micelle & pDNA & N/A & N/A & N/A & [68] \\
\hline DMEDA-HPbCD-Chol:Pluronic F127 & \multirow{3}{*}{ polyplex } & \multirow{3}{*}{ siRNA } & \multirow{3}{*}{ N/A } & \multirow{3}{*}{ N/A } & \multirow{3}{*}{ N/A } & \multirow{3}{*}{ [118] } \\
\hline DMEDA-HPbCD-Chol:Pluronic L81 & & & & & & \\
\hline DMEDA-HPbCD-Chol:Pluronic L35 & & & & & & \\
\hline PAMAM-Chol & micelle & RES & N/A & N/A & 46.5 & [119] \\
\hline \multirow{4}{*}{ PEG-Chol- $\alpha-$ TOC } & \multirow{4}{*}{ micelle } & CUR $(5 \% w / w)$ & \multirow{4}{*}{ N/A } & 97.2 & 4.6 & \multirow{4}{*}{ [69] } \\
\hline & & CUR $(10 \% w / w)$ & & 98.4 & 8.4 & \\
\hline & & CUR $(15 \% w / w)$ & & 98.6 & 14.2 & \\
\hline & & CUR $(20 \% w / w)$ & & 74.3 & 15.2 & \\
\hline mPEG- $b$-PEP- $g$-Chol) linear & \multirow{3}{*}{ micelle } & \multirow{3}{*}{ DOX } & $5.8(1.45)$ & 42.1 & 15.7 & \multirow{3}{*}{ [70] } \\
\hline mPEG- $b$-PEP- $g$-Chol) Y-shape & & & $6.0(1.33)$ & 50.4 & 20.2 & \\
\hline mPEG- $b$-PEP- $g$-Chol) Fork-shape & & & $6.5(1.48)$ & 58.5 & 23.1 & \\
\hline \multirow{3}{*}{ mPEG-b-PAMAM-G ${ }_{1}$ - $\mathrm{Chol}_{1}$} & \multirow{12}{*}{ micelle } & DOX & \multirow{3}{*}{$5.3(\mathrm{~N} / \mathrm{A})$} & 40.0 & 4.7 & \multirow{12}{*}{ [71] } \\
\hline & & PTX & & 5.4 & 0.7 & \\
\hline & & DOX/PTX & & $38.1 / 6.5$ & $4.3 / 0.7$ & \\
\hline \multirow{3}{*}{ mPEG- $b$-PAMAM-G ${ }_{2}-\mathrm{Chol}_{2}$} & & DOX & \multirow{3}{*}{5.9 (N/A) } & 40.7 & 4.8 & \\
\hline & & PTX & & 7.2 & 0.8 & \\
\hline & & DOX/PTX & & $38.4 / 6.9$ & $4.2 / 0.8$ & \\
\hline \multirow{3}{*}{ mPEG-b-PAMAM-G ${ }_{4}-\mathrm{Chol}_{4}$} & & DOX & \multirow{3}{*}{$7.2(\mathrm{~N} / \mathrm{A})$} & 40.2 & 4.8 & \\
\hline & & PTX & & 8.3 & 1.0 & \\
\hline & & DOX/PTX & & $34.5 / 8.3$ & $3.9 / 1.0$ & \\
\hline \multirow{3}{*}{ mPEG- $b$-PAMAM-G ${ }_{8}-\mathrm{Chol}_{8}$} & & DOX & \multirow{3}{*}{ N/A } & 40.1 & 4.7 & \\
\hline & & PTX & & 18.2 & 2.2 & \\
\hline & & DOX/PTX & & $36.8 / 19.4$ & $4.2 / 2.2$ & \\
\hline Chol-P(HEMA-Lys) & liposome & siRNA & N/A (1.20) & N/A & N/A & [120] \\
\hline mPEG-P(HPMA-g-His)-Chol & liposome & DOX & $12.3(1.06)$ & 81.3 & 18.2 & {$[25,26]$} \\
\hline
\end{tabular}


Table 2. Cont.

\begin{tabular}{|c|c|c|c|c|c|c|}
\hline Polymer & Form of Carrier & Drug or Dye & $M_{n}$ or $M_{w}(k D a)(Đ)$ & LE (\%) & LC (\%) & Lit. \\
\hline \multirow{2}{*}{ (Chol-PLGVRK-PEG):(DUPA-PEG-Chol) = 1:9 } & \multirow{2}{*}{ micelle } & CABA $(25 \% w / w)$ & \multirow{2}{*}{ N/A } & 79.7 & 12.0 & \multirow{2}{*}{ [72] } \\
\hline & & CABA $(200 \% w / w)$ & & 38.9 & 43.8 & \\
\hline Chol-g-uPA-PAA & liposome & $\mathrm{CF}$ & N/A & N/A & N/A & {$[121]$} \\
\hline \multirow{4}{*}{ CS- $g$-Chol- $g$-FA } & \multirow{4}{*}{ micelle } & PTX (4h dialysis) & \multirow{4}{*}{ N/A } & 75.6 & 12.9 & \multirow{4}{*}{ [11] } \\
\hline & & PTX (8h dialysis) & & 63.1 & 10.5 & \\
\hline & & PTX (12h dialysis) & & 56.5 & 7.4 & \\
\hline & & PTX (24h dialysis) & & 32.7 & 5.5 & \\
\hline Chol-DP7 & micelle & itself & N/A & N/A & $\mathrm{N} / \mathrm{A}$ & [122] \\
\hline \multicolumn{7}{|c|}{ Radical Cross-Coupling } \\
\hline $\mathrm{p}$ (HPMA-r-NAS)-Chol & \multirow{3}{*}{ polyplex } & \multirow{3}{*}{ siRNA } & $17.7(1.40)$ & \multirow{3}{*}{$\mathrm{N} / \mathrm{A}$} & \multirow{3}{*}{ N/A } & \multirow{3}{*}{ [27] } \\
\hline p(HPMA-r-AEDA)-Chol & & & $24.7(1.20)$ & & & \\
\hline p(HPMA-DMAE-r-AEDA)-Chol & & & $34.1(1.30)$ & & & \\
\hline \multicolumn{7}{|c|}{ Hydrazone Formation } \\
\hline \multirow{2}{*}{ mPEG-Hz-Chol } & \multirow{2}{*}{ liposome } & Arctigenin & N/A & 93.8 & N/A & [73] \\
\hline & & GEM & $2.6(\mathrm{~N} / \mathrm{A})$ & 37.0 & 4.0 & {$[74,75]$} \\
\hline
\end{tabular}

Abbreviations: AEDA, 2-((2-azidoethyl) disulfanyl) ethan-1-amine hydrochloride; AmB, Amphotericin B; CABA, cabazitaxel; Chol, cholesterol; CF, 5,6-carboxyfluorescein; CS, chitosan; CUR, curcumin; CYS, cystamine; DMAE, 2-(dimethylamino)ethyl 1H-imidazole-1-carboxylate; DMAAm, $N, N$-dimethylacrylamide; DMEDA, $N, N$-dimethylaminoethylamine; pDNA, plasmid DNA; DOX, doxorubicin; DP7, antimicrobial peptide (VQWRIRVAVIRK); DTXL, docetaxel; DUP1, peptide (CFRPNRAQDYNTN); DUPA, 2-[3-(1,3-dicarboxypropyl) ureido]pentanedioic acid; F68, Pluronic F68; FA, folic acid; Fmoc, 9-fluorenylmethoxycarbonyl; GA, glutamic acid; GEM, gemcitabine; HA, hyaluronic acid; (HE) 5 , histidine-glutamic acid decapeptide; HEMA, hydroxyethyl methylacrylate; HIS, histidine; HPbCD, modified 2-hydroxypropyl-b-cyclodextrin macrocycles; HPMA, N-(2-hydroxypropyl) methacrylamide; Hz, hydrazone; ICG, indocyanine green; Lys, lysine; mPEG, (poly(ethylene glycol) methylether methacrylate; NAS, $N$-acryloxysuccinimide; NIPAAm, $N$-isopropylacrylamide; PAA, poly(acrylic acid); PAE, poly( $\beta$-amino ester); PAMAM, polyamidoamine; PAsp(DET), poly $\{N$-[N-(2-aminoethyl)-2-aminoethyl] aspartamide $\} ; \mathrm{Pbf}, 2,2,4,6,7$-pentamethyldihydrobenzofuran-5-sulfonyl; PCL, poly( $\varepsilon$-caprolactone); PDLA, poly(D-lactide acid); PEG, poly(ethylene glycol); PEP, peptide; PF127, Synperonic PE/F 127; PGA, poly(glutamic acid); PLA, poly(lactic acid); PLGA, PCL, poly(E-caprolactone); PDLA, poly(D-lactide acid); PEG, poly(ethylene glycol); PEP, peptide; PF127, Synperonic PE/F 127; PGA, poly(glutamic acid); PLA, poly(lactic acid); PLGA,
poly(D,L-lactide-co-glycolide); PLGVRK, matrix metalloproteinase-2 responsive peptide; PLLA, poly(L-lactide acid); PplX, protoporphyrin IX; PSO, polyoxyethylene sorbitol oleate; PTX, paclitaxel; Py, pyrene; QC, quercetin; RES, resveratrol; (RG) $)_{5}$, arginine-glycine decapeptide; RGD, arginylglycylaspartic acid; SA, succinic anhydride; TMX, tamoxifen; TPGS, tocopheryl poly(ethylene glycol) succinate; TPL, triptolide; TPP, triphenylphosphine; $\alpha$-TOC, $\alpha$-tocopherol; uPA, short peptide sequence for urokinase plasminogen activator. 
The introduction of cholesterol at the end of the polymer chain by post-modification occurs mainly through basic organic chemistry reactions such as esterification and amidation (Table 2). In this case, the simpler the better, thus reducing the time, cost, and risk of failure. The esterification of the $\mathrm{OH}$-terminated polymer in dichloromethane in the presence of 4-dimethylaminopyridine (DMAP) with commercially available cholesteryl chloroformate and triethylamine is often used [58, $112,114,116]$. Reactions with succinyl cholesterol have been also reported, and, in these cases, dicyclohexylcarbodiimide (DCC) or 1-(3-dimethylaminopropyl)-3-ethylcarbodiimide (EDC) was used $[56,57,59]$. Amidation is mainly based on EDC coupling, where the NHS ester derivative of cholesterol or polymer is dissolved in an organic solvent (DCM, MeOH, or DMSO); then, in the presence of EDC, it reacts with a previously prepared amine-terminated derivative of a polymer or cholesterol, respectively $[25,26,67,69,72,120]$. There is also the possibility to introduce cholesterol in a radical cross-coupling [27] reaction or by hydrazone formation [73-75].

\subsection{Polymers Containing Cholesterol as Side Chains}

Similarly to the polymers containing cholesterol in the main chain, the incorporation of cholesterol to the side chains can occur by polymerization of a cholesterol-containing monomer (Figure 5, Table 3) or by post-polymerization modification (Figure 6, Table 4). Compared to cholesterol end-capped polymers, this approach allows the incorporation of multiple cholesterol molecules into a single polymeric chain. However, it carries a large ballast of the hydrophobic part, which has a negative effect on aqueous solubility. As a consequence, it creates the need to extend or add a new hydrophilic block (most often PEG), which in turn increases the weight of the carrier introduced into the body.

\subsubsection{Polymers Containing Cholesterol Moieties as Side Chains Obtained by Polymerization of} Cholesterol-Based Monomers

Many different polymerization techniques are used to obtain polymeric drug carriers with cholesterol moieties as side chains, ranging from free radical polymerization to various types of controlled polymerization methods, such as RAFT, ATRP, and a variety of ring-opening polymerization methods such as ring-opening metathesis polymerization (ROMP) and organocatalytic ring-opening polymerization (OC-ROP) (Figure 5, Table 3). The variation of the methods used is due to many factors. One, as in all areas of life, is economics, i.e., the method should be non-expensive, technically simple, limit the use of toxic chemicals and give a clean product with high efficiency. However, in the case of DDS, it is not easy to achieve, because products that have a complex spatial structure and consist of many block-elements are considered. Drug delivery systems, due to their destination-the human body - should be characterized as accurately as possible. The more monodisperse sample, the more accurate its properties and expected behavior in the body. In the case of polymeric systems characterized by high dispersity, it is almost impossible to conclude the mechanism of action, metabolism, or removal. Therefore, the controlled polymerization techniques, which allow precise designing of polymers of desired molecular weight (number of repeating units), spatial structure, and low dispersion, are the methods of choice.

Similar copolymers of HPMA and various methacrylic cholesterol derivatives have been obtained by FRP or RAFT. The copolymer obtained by the controlled polymerization was characterized by significantly lower dispersion (1.39) [23] than the analogous copolymers produced by FRP (1.65-1.90) [19-22], despite the weight being approximately twice as high. The most important feature of ROPs is the ability to polymerize functionalized cyclic olefins [96]. However, it also carries a toxic ballast in the form of initiators or catalysts based on transition metals such as tin, ruthenium, or molybdenum in the ROMP [96]. 


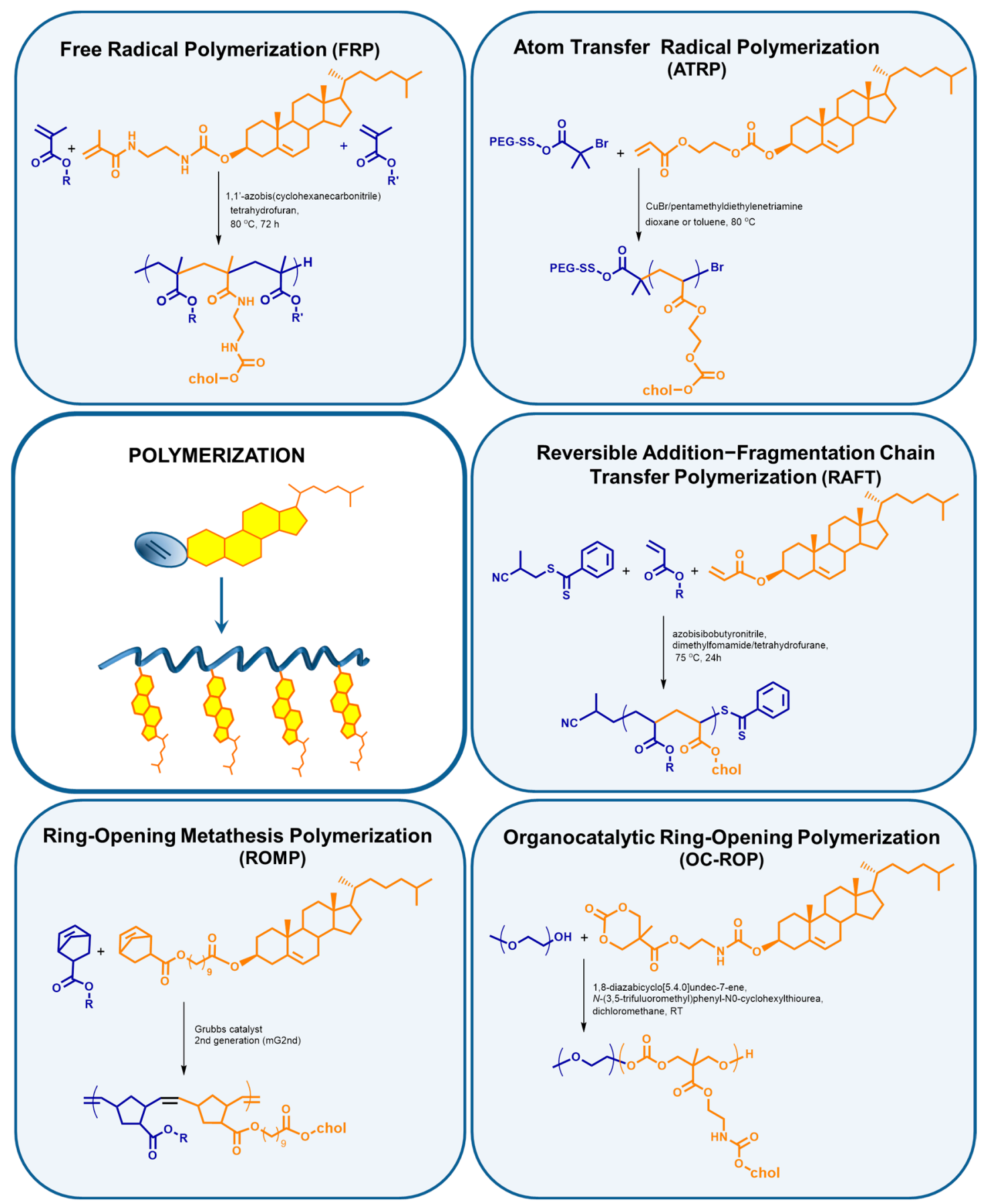

Figure 5. Types of polymerization used to obtain polymers with cholesterol moieties as side chains, and reaction examples [30,32,34-36]. 
Table 3. Polymers bearing cholesterol in side chains reported as drug delivery systems.

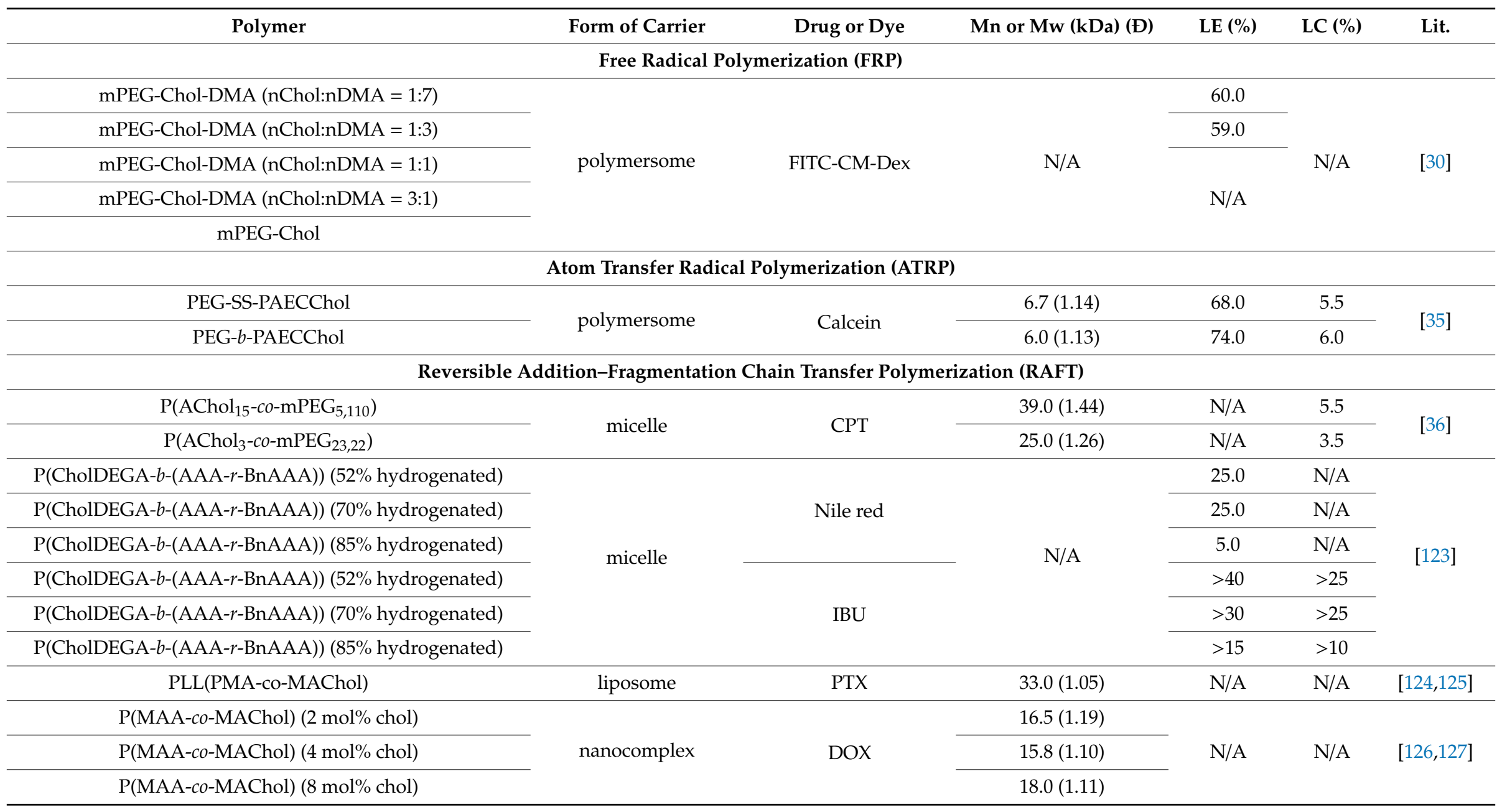


Table 3. Cont

\begin{tabular}{|c|c|c|c|c|c|c|}
\hline Polymer & Form of Carrier & Drug or Dye & Mn or Mw (kDa) (Đ) & LE (\%) & LC (\%) & Lit. \\
\hline P(MAgala $18-b-$ MAChol $\left._{14}\right)$ & \multirow{4}{*}{ micelle } & \multirow{4}{*}{ DOX } & $12.8(1.26)$ & 47.1 & 10.5 & \multirow{4}{*}{ [128] } \\
\hline P(MAgala $\left.18-b-\left(\mathrm{MAA}_{5}-\mathrm{co}-\mathrm{MAChol}_{14}\right)\right)$ & & & \multirow{3}{*}{ N/A } & 61.5 & 13.3 & \\
\hline $\mathrm{P}\left(\mathrm{MAgala}_{18}-b-\left(\mathrm{MAA}_{16}-\mathrm{co}-\mathrm{MAChol}_{12}\right)\right)$ & & & & 81.9 & 17.0 & \\
\hline P(MAgala $18^{\left.-b-\left(\mathrm{MAA}_{26}-\mathrm{co}-\mathrm{MAChol}_{9}\right)\right)}$ & & & & 91.2 & 18.6 & \\
\hline P(HPMA-co-MA- $\varepsilon$ Ahx-NHNH $\left.{ }_{2}-c o-M A-\varepsilon A h x-C h o l\right)$ & nanoparticle & DOX & $50(1.39)$ & $\mathrm{N} / \mathrm{A}$ & 6.0 & [23] \\
\hline \multicolumn{7}{|c|}{ Organocatalytic Ring-Opening Polymerization (OC-ROP) } \\
\hline $\mathrm{mPEG}_{113}-b-\mathrm{P}(\mathrm{MTC}-\mathrm{Chol})_{4}$ & \multirow{2}{*}{ micelle } & \multirow{2}{*}{ N/A } & $7.5(1.12)$ & \multirow{2}{*}{ N/A } & \multirow{2}{*}{ N/A } & \multirow{2}{*}{ [31] } \\
\hline $\mathrm{mPEG}_{113}-b-\mathrm{P}(\mathrm{MTC}-\mathrm{Chol})_{11}$ & & & $11.8(1.21)$ & & & \\
\hline $\mathrm{mPEG}_{113}-b-\mathrm{P}\left(\mathrm{MTC}-\mathrm{Chol}_{11}\right)$ & \multirow{4}{*}{ nanoparticle } & \multirow{4}{*}{ PTX } & $11.8(1.21)$ & \multirow{4}{*}{ N/A } & 3.8 & \multirow{4}{*}{ [32] } \\
\hline $\mathrm{mPEG}_{113}-b-\mathrm{P}\left(\mathrm{MTC}-\mathrm{Chol}_{8}-\mathrm{co}-\mathrm{TMC}_{8}\right)$ & & & $10.7(1.18)$ & & 9.2 & \\
\hline $\mathrm{mPEG}_{113}-b-\mathrm{P}\left(\mathrm{MTC}-\mathrm{Chol}_{11}-c o-\mathrm{TMC}_{30}\right)$ & & & $14.8(1.20)$ & & 15.0 & \\
\hline $\mathrm{mPEG}_{113}-b-\mathrm{P}\left(\mathrm{MTC}-\mathrm{Chol}_{18}-\mathrm{co}-\mathrm{TMC}_{55}\right)$ & & & $21.7(1.17)$ & & 8.4 & \\
\hline \multicolumn{7}{|c|}{ Ring-Opening Metathesis Polymerization (ROMP) } \\
\hline P(NBChol- $b$-NBmPEG) & nanoparticle & DOX & $162(1.30)$ & 58.0 & 14.5 & [33] \\
\hline $\mathrm{P}(\mathrm{NBChol})_{50}-b-(\mathrm{NBmPEG})_{170}$ & \multirow{3}{*}{ nanoparticle } & \multirow{3}{*}{ DOX } & $126(1.24)$ & 88.4 & 22.1 & \multirow{3}{*}{ [34] } \\
\hline $\mathrm{P}(\mathrm{NBChol})_{75}-b-(\mathrm{NBmPEG})_{255}$ & & & $216(1.16)$ & 68.8 & 17.2 & \\
\hline $\mathrm{P}(\mathrm{NBChol})_{180^{-} b-(\mathrm{NBmPEG})_{222}}$ & & & $118(1.16)$ & 79.2 & 19.8 & \\
\hline
\end{tabular}

Abbreviations: AAA, ascorbyl acrylate; AChol, cholesteryl acrylate; AECChol, cholesteryl acryloyoxy ethyl carbonate; ATRP, atom transfer radical polymerization; BnAAA, benzyl protected ascorbylacrylate; Chol, cholesterol; CholDEGA, cholesteryl diethyleneglycol acrylate; CPT, S-(+)-camptothecin; DMA, 1-decyl methacrylate; DOX, doxorubicin; DTXL, docetaxel; FITC-CM-Dex, fluorescein isothiocyanate carboxymethyl dextran; HIS, histidine; HPMA, N-(2-hydroxypropyl)methacrylamide; IBU, ibuprofen; MAA, methacrylic acid;

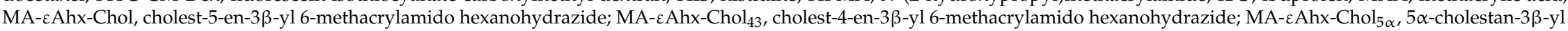

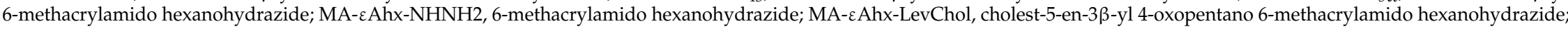
MA- $\varepsilon$ Ahx-opB-Chol, cholest-5-en-3 $\beta$-yl-4-(2-oxopropyl)-benzoate; MAChol, 6-cholesteryloxyhexyl methacrylate; MAgala, 6-Omethacryloyl-D-galactopyranose; mPEG, (poly(ethylene glycol) methylether methacrylate; MTC-Chol, cholesteryl 2-(5-methyl-2-oxo-1;3-dioxane-5-carboxyloyloxy)ethyl carbamate); NB, norbornene; OC-ROP, organocatalytic ring-opening polymerization; PAE, poly( $\beta$-amino ester); PEG, poly(ethylene glycol); PLL, poly(L-lysine); PTX, paclitaxel; RAFT, reversible addition-fragmentation chain transfer polymerization; ROMP, ring-opening metathesis polymerization; $\mathrm{SS}$, disulfide bridge; TMC, trimethylene carbonate. 


\subsubsection{Polymers Bearing Cholesterol Moieties as Side Chains Obtained by Post-Modification}

In addition to the classical amidation or esterification methods, which require the use of catalysts such as 1-(3-dimethylaminopropyl)-3-ethylcarbodiimide (EDC), dicyclohexylcarbodiimide (DCC), 4-dimethylaminopyridine (DMAP), or 1,8-diazabicyklo[5.4.0]undek-7-en (DBU), there are increasing possibilities of functionalization of polymers through post-modification, growing with the development of organic chemistry. The use of polymers in the drug delivery process requires the highest purity of polymer systems. Toxic catalysts and solvents, or complex and time- and cost-consuming purification processes force scientists to create new synthetic methods that take place under milder conditions. Alternative methods such as supercritical $\mathrm{CO}_{2}$-assisted spray drying (SASD) [80], nucleophilic substitution (Br to N) [129-131] or N-acylation [132] are gaining popularity due to the lack of catalysts and simple isolation and purification of reaction products (Figure 6, Table 4).

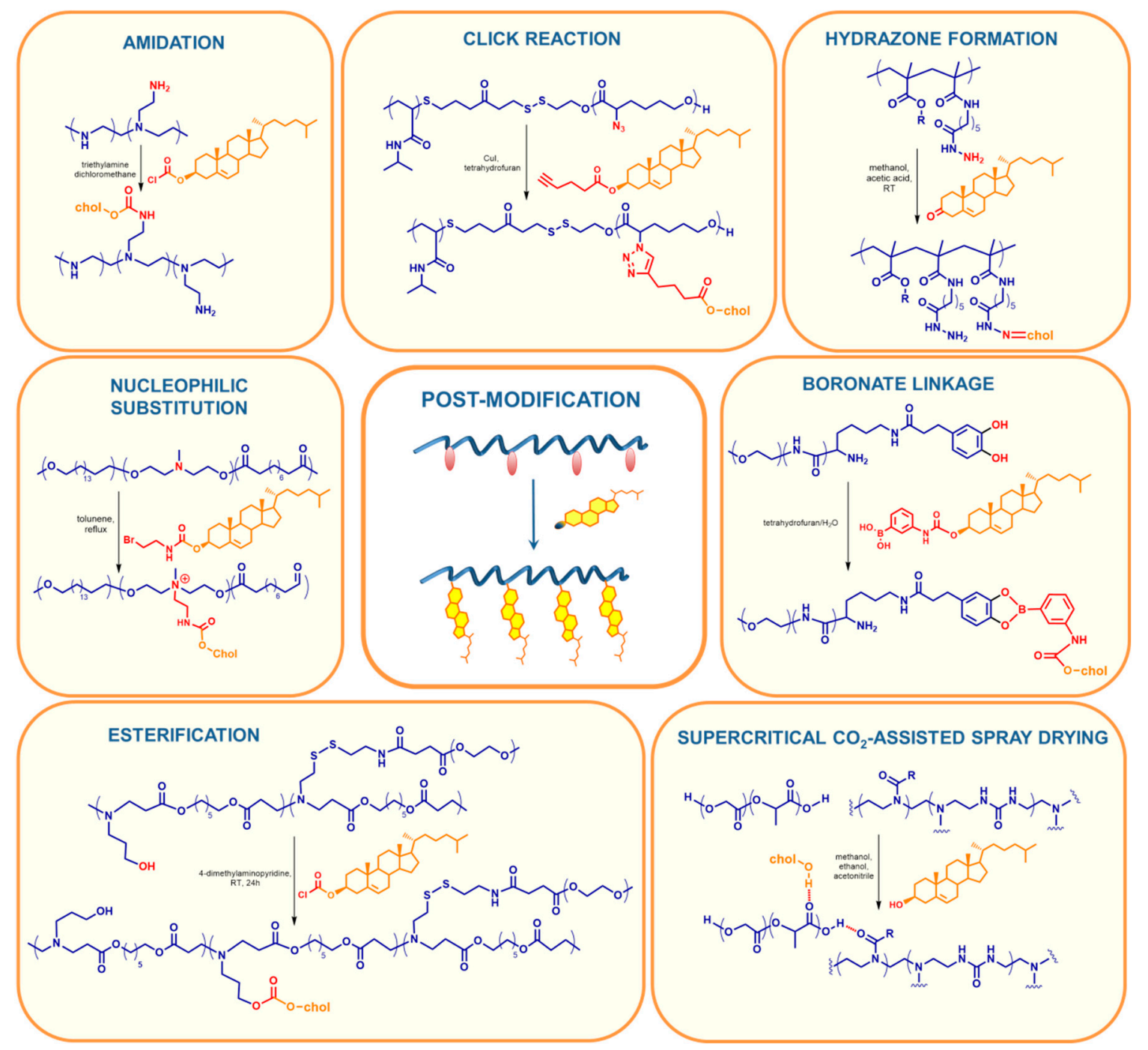

Figure 6. Methods of incorporation of cholesteryl moieties to side chains by post-polymerization reactions $[20,38,39,80,130,132,133]$. 
Table 4. Polymers containing cholesterol moieties in side chains introduced by post-modification.

\begin{tabular}{|c|c|c|c|c|c|c|c|}
\hline Polymer & Form of Carrier & Drug or Dye & Mn or Mw (kDa) (Đ) & LE (\%) & LC $(\%)$ & $\operatorname{Dg}(\%)$ & Lit. \\
\hline \multicolumn{8}{|c|}{ Amidation } \\
\hline PEI-Chol & liposome & $\begin{array}{l}\text { pDNA (pGL3 } \\
\text { promoter) }\end{array}$ & N/A & N/A & $\mathrm{N} / \mathrm{A}$ & N/A & [134] \\
\hline \multirow{5}{*}{ HA-Chol } & \multirow{5}{*}{ nanogel } & \multirow{5}{*}{$\begin{array}{c}\mathrm{rhGH} / \mathrm{EPO} \\
\text { lysozyme/exendin-4 }\end{array}$} & $52.0(\mathrm{~N} / \mathrm{A})$ & \multirow{5}{*}{$\mathrm{N} / \mathrm{A}$} & \multirow{5}{*}{ N/A } & 3.0 & \multirow{5}{*}{ [14] } \\
\hline & & & $54.0(\mathrm{~N} / \mathrm{A})$ & & & 7.0 & \\
\hline & & & $59.0(\mathrm{~N} / \mathrm{A})$ & & & 15.0 & \\
\hline & & & 66.0 (N/A) & & & 27.0 & \\
\hline & & & 75.0 (N/A) & & & 42.0 & \\
\hline P(MPC-co-NPEM)-g-Chol (nMPC:nChol = 86:14) & \multirow{2}{*}{ micelle } & \multirow{2}{*}{ DOX } & $11.2(1.95)$ & 53.8 & 21.5 & \multirow{9}{*}{100} & \multirow{2}{*}{ [135] } \\
\hline P(MPC-co-NPEM)-g-Chol-g-FA (nMPC:nChol:nFA = 56:14:30) & & & $12.1(2.16)$ & 53.5 & 21.4 & & \\
\hline P(MPC-co-NPEM)- $g$-Chol- $g$-FA (nMPC:nChol = 74:26) & \multirow{7}{*}{ micelle } & & $6.6(\mathrm{~N} / \mathrm{A})$ & 48.6 & 19.6 & & \multirow{7}{*}{ [136] } \\
\hline P(MPC-co-NPEM)-g-Chol- $g$-FA (nMPC:nChol:nFA = 64:27:9) & & DOX & \multirow{5}{*}{$\mathrm{N} / \mathrm{A}$} & 52.6 & 21.1 & & \\
\hline \multirow{4}{*}{ P(MPC-co-NPEM)- $g$-Chol- $g$-FA (nMPC:nChol:nFA = 61:16:23) } & & $\operatorname{DOX}(10 \% w / w)$ & & 81.9 & 8.2 & & \\
\hline & & $\operatorname{DOX}(20 \% w / w)$ & & 77.9 & 15.6 & & \\
\hline & & $\operatorname{DOX}(40 \% w / w)$ & & 62.3 & 24.9 & & \\
\hline & & $\operatorname{DOX}(50 \% w / w)$ & & 67.4 & 33.7 & & \\
\hline P(MPC-co-NPEM)- $g$-Chol- $g$-FA (nMPC:nChol:nFA = 58:11:31) & & DOX & $11.4(\mathrm{~N} / \mathrm{A})$ & 58.8 & 23.5 & & \\
\hline$\gamma$-PGA-g-Chol & hydrogel & DOX & N/A & N/A & 6.39 & 96.2 & [76] \\
\hline PEI-Chol & polyplex & siRNA & $\mathrm{N} / \mathrm{A}$ & N/A & N/A & N/A & $\begin{array}{l}{[137,} \\
138]\end{array}$ \\
\hline PEI-Chol (nPEI:nChol = 1:7.5) & \multirow{4}{*}{ micelle } & \multirow{4}{*}{ SFB } & $9.8(\mathrm{~N} / \mathrm{A})$ & \multirow{4}{*}{$\mathrm{N} / \mathrm{A}$} & N/A & \multirow{4}{*}{$\mathrm{N} / \mathrm{A}$} & \multirow{4}{*}{ [37] } \\
\hline PEI-Chol (nPEI:nChol = 1:15.5) & & & $13.1(\mathrm{~N} / \mathrm{A})$ & & 13.1 & & \\
\hline PEI-Chol-PEG (nPEI:nChol:nPEG = 1:7.5:1) & & & $15.3(\mathrm{~N} / \mathrm{A})$ & & N/A & & \\
\hline PEI-Chol-PEG (nPEI:nChol:nPEG = 1:15.5:1) & & & 23.9 (N/A) & & N/A & & \\
\hline Chol-CA-Spe & nanogel & siRNA & $\mathrm{N} / \mathrm{A}$ & N/A & N/A & 3.1 & [139] \\
\hline Cyc-PEI-Chol (nChol:nCyc = 0.17) & \multirow{3}{*}{ polyplex } & \multirow{3}{*}{ siRNA } & 28.8 (N/A) & \multirow{3}{*}{$\mathrm{N} / \mathrm{A}$} & \multirow{3}{*}{ N/A } & \multirow{3}{*}{ N/A } & \multirow{3}{*}{ [140] } \\
\hline Cyc-PEI-Chol (nChol:nCyc = 0.33) & & & $32.4(\mathrm{~N} / \mathrm{A})$ & & & & \\
\hline Cyc-PEI-Chol (nChol:nCyc = 0.53) & & & 36.9 (N/A) & & & & \\
\hline
\end{tabular}


Table 4. Cont

\begin{tabular}{|c|c|c|c|c|c|c|c|}
\hline Polymer & Form of Carrier & Drug or Dye & Mn or Mw (kDa) (Đ) & LE (\%) & LC (\%) & $\operatorname{Dg}(\%)$ & Lit. \\
\hline PEI-Chol & nanoparticle & Ce6 & $\mathrm{N} / \mathrm{A}$ & N/A & 35 & N/A & [141] \\
\hline Chol-GC & \multirow{8}{*}{ micelle } & \multirow{4}{*}{ DOX } & \multirow{8}{*}{$\mathrm{N} / \mathrm{A}$} & 80.9 & 10.8 & \multirow{8}{*}{6.1} & \multirow{8}{*}[142]{} \\
\hline Chol-GC-FA & & & & 87.0 & 11.6 & & \\
\hline NLS-Chol-GC & & & & 77.4 & 10.4 & & \\
\hline NLS-Chol-GC-FA & & & & 79.0 & 10.6 & & \\
\hline Chol-GC & & \multirow{4}{*}{ Cou6 } & & 89.8 & 1.76 & & \\
\hline Chol-GC-FA & & & & 87.0 & 1.71 & & \\
\hline NLS-Chol-GC & & & & 90.1 & 1.77 & & \\
\hline NLS-Chol-GC-FA & & & & 89.6 & 1.73 & & \\
\hline PEI-Chol (nChol:nPEI = 25.8) & \multirow{6}{*}{ polyplex } & \multirow{6}{*}{ siRNA } & \multirow{6}{*}{ N/A } & \multirow{6}{*}{ N/A } & \multirow{6}{*}{ N/A } & \multirow{6}{*}{ N/A } & \multirow{6}{*}{143} \\
\hline PEI-Chol (nChol:nPEI = 52.5) & & & & & & & \\
\hline PEI-Chol (nChol:nPEI = 102.44) & & & & & & & \\
\hline F-PEI-Chol (nChol:nF-PEI = 21.3) & & & & & & & \\
\hline F-PEI-Chol (nChol:nF-PEI = 50.9) & & & & & & & \\
\hline F-PEI-Chol (nChol:nF-PEI = 105.6) & & & & & & & \\
\hline PAMD-Chol $(17 \% w / w$ of Chol) & \multirow{3}{*}{ polyplex } & \multirow{3}{*}{ siRNA } & 16.7 & \multirow{3}{*}{ N/A } & \multirow{3}{*}{ N/A } & \multirow{3}{*}{ N/A } & \multirow{3}{*}{144} \\
\hline PAMD-Chol $(25 \% w / w$ of Chol) & & & 18.5 & & & & \\
\hline PAMD-Chol $(34 \% w / w$ of Chol) & & & 21.1 & & & & \\
\hline PEI-Chol & N/A & siRNA & N/A & N/A & N/A & N/A & [132] \\
\hline \multicolumn{8}{|c|}{ Click Reaction } \\
\hline PNIPAAm $10-\mathrm{SS}-\mathrm{P}\left(\alpha \mathrm{N}_{3} \mathrm{CL}-\mathrm{g} \text {-CholPA }\right)_{10}$ & \multirow{2}{*}{ micelle } & \multirow{2}{*}{ IMC } & $6.0(1.24)$ & 82.8 & 40.4 & \multirow{2}{*}{$\mathrm{N} / \mathrm{A}$} & \multirow{2}{*}{ [133] } \\
\hline PNIPAAm $_{10}-\mathrm{SS}-\mathrm{P}\left(\alpha \mathrm{N}_{3} \mathrm{CL}_{10}-g\right.$-PyrePA $\left.3 /-\mathrm{CholPA}_{7}\right)$ & & & $5.7(1.40)$ & 71.9 & 35.9 & & \\
\hline acL-Chol-PN & \multirow{2}{*}{ nanogel } & \multirow{2}{*}{ FITC-BSA } & 1020 (N/A) & \multirow{2}{*}{ N/A } & \multirow{2}{*}{ N/A } & 1.7 & \multirow{2}{*}{ [145] } \\
\hline acS-Chol-PN & & & 1130 (N/A) & & & 1.5 & \\
\hline \multicolumn{8}{|c|}{ Hydrazone Formation } \\
\hline \multirow{2}{*}{ P(HPMA-co-MA- $\varepsilon$ Ahx-NHNH ${ }_{2}$-co-MA- $\varepsilon$ Ahx-Chol) } & nanoparticle & DOX & 211 (165) & 98.0 & 1.7 & & [19] \\
\hline & Hanoparticte & DTXL & $21.1(1.00)$ & 95.0 & 5.5 & & 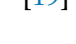 \\
\hline
\end{tabular}


Table 4. Cont

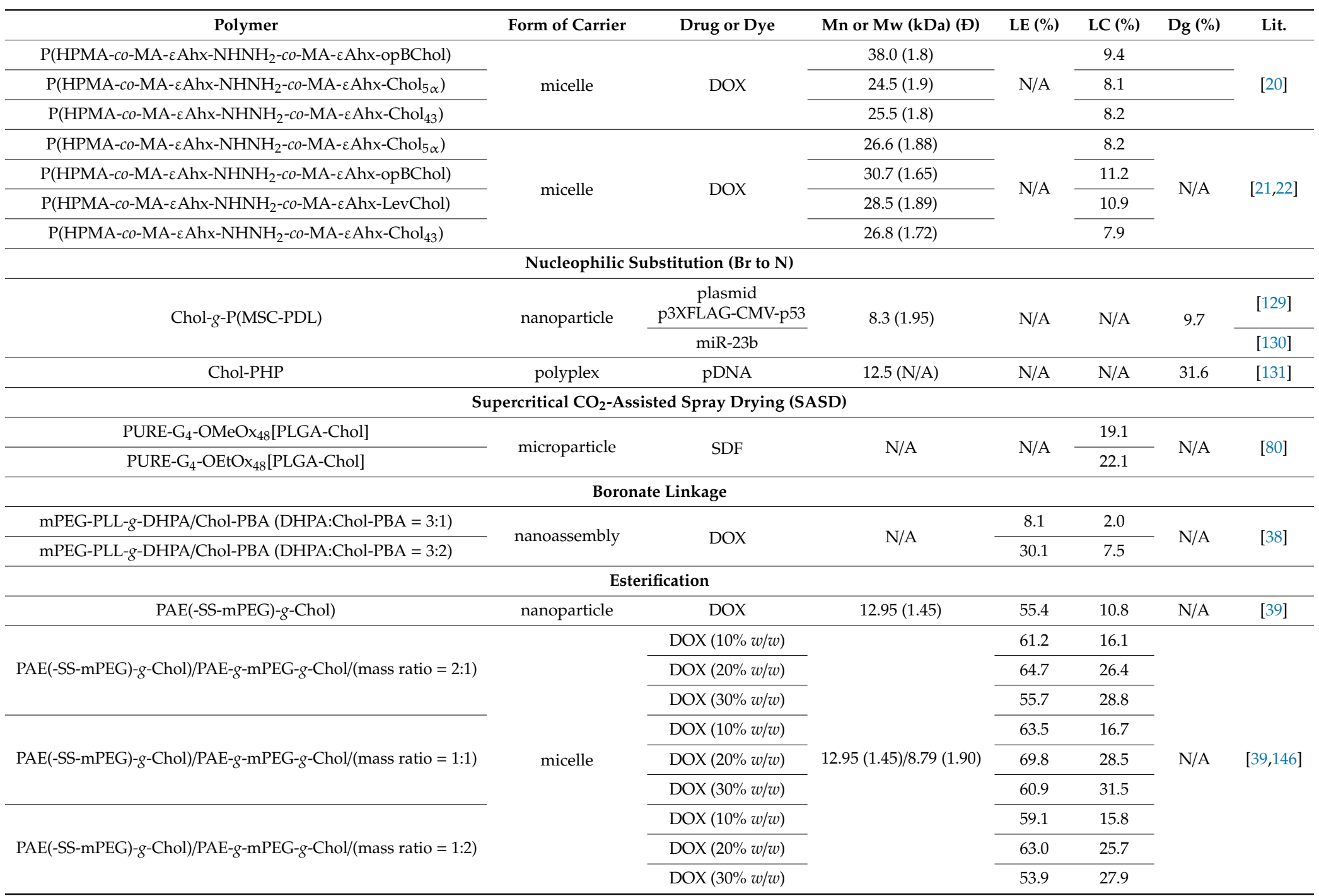


Table 4. Cont.

\begin{tabular}{|c|c|c|c|c|c|c|c|}
\hline Polymer & Form of Carrier & Drug or Dye & Mn or Mw (kDa) (Đ) & LE (\%) & LC (\%) & $\operatorname{Dg}(\%)$ & Lit. \\
\hline poly(BAC-AMPD)- $g$-PEG- $g$-Chol & micelle & DOX & N/A & 27.1 & 5.4 & 54.5 & [40] \\
\hline \multirow{4}{*}{ Chol-CS } & \multirow{4}{*}{ nanoparticle } & ATRA $(10 \% w / w)$ & \multirow{4}{*}{ N/A } & 88.7 & 8.0 & \multirow{4}{*}{4} & \multirow{4}{*}{ [12] } \\
\hline & & ATRA $(20 \% w / w)$ & & 82.3 & 11.8 & & \\
\hline & & ATRA $(40 \% w / w)$ & & 77.9 & 24.3 & & \\
\hline & & ATRA $(50 \% w / w)$ & & 74.0 & 28.3 & & \\
\hline \multirow{4}{*}{ rPAA-Chol } & \multirow{4}{*}{ nanoparticle } & \multirow{4}{*}{ siRNA } & 9.7 (N/A) & \multirow{4}{*}{ N/A } & \multirow{4}{*}{ N/A } & 14.0 & \multirow{4}{*}{$\begin{array}{l}{[147} \\
148]\end{array}$} \\
\hline & & & 10.9 (N/A) & & & 29.0 & \\
\hline & & & $13.5(\mathrm{~N} / \mathrm{A})$ & & & 57.0 & \\
\hline & & & 15.9 (N/A) & & & 87.0 & \\
\hline \multirow{4}{*}{ (PAE- $g$-Chol)- $b$-PEG- $b$-(PAE- $g$-Chol) } & \multirow{4}{*}{ micelle } & $\operatorname{DOX}(10 \% w / w)$ & \multirow{4}{*}{ N/A } & 33.6 & 4.2 & \multirow{4}{*}{48.0} & \multirow{4}{*}{ [41] } \\
\hline & & $\operatorname{DOX}(20 \% w / w)$ & & 48.7 & 13.5 & & \\
\hline & & $\operatorname{DOX}(50 \% w / w)$ & & 59.5 & 20.1 & & \\
\hline & & $\operatorname{DOX}(80 \% w / w)$ & & 55.3 & 24.3 & & \\
\hline PEG-PMMI-CholC6 & liposome & RAPA & $74.0(1.51)$ & 76.9 & N/A & 4.9 & [42] \\
\hline PEG-PMMI-CholC6 & liposome & MTX & N/A & 63.1 & N/A & N/A & [43] \\
\hline PMMI-CholC6 & \multirow{2}{*}{ micelle } & \multirow{2}{*}{$\mathrm{PX}$} & $57.1(1.60)$ & 30.0 & 6.2 & 4.9 & \multirow{2}{*}{ [44] } \\
\hline PEG-PMMI-CholC6 & & & $74.3(1.51)$ & 40.3 & 8.3 & 16.4 & \\
\hline \multirow{2}{*}{ Chol-PEG $22-$ hbPG $_{35}$} & \multirow{2}{*}{ liposome } & Atto 488 tetrazine & \multirow{2}{*}{ N/A } & $>40$ & \multirow{2}{*}{ N/A } & \multirow{2}{*}{ N/A } & \multirow{2}{*}{ [45] } \\
\hline & & $\begin{array}{l}\text { Alexa Fluor } 594 \\
\text { azide }\end{array}$ & & $>40$ & & & \\
\hline \multirow{3}{*}{ HA-Chol } & \multirow{3}{*}{ micelle } & $\alpha-\mathrm{TOC}$ & \multirow{3}{*}{ N/A } & 77.6 & 16.1 & \multirow{3}{*}{4.6} & \multirow{3}{*}{ [15] } \\
\hline & & CUR & & 82.8 & 3.3 & & \\
\hline & & CoQ10 & & 86.2 & 10.7 & & \\
\hline
\end{tabular}


Table 4. Cont.

\begin{tabular}{|c|c|c|c|c|c|c|c|}
\hline Polymer & Form of Carrier & Drug or Dye & Mn or Mw (kDa) (Đ) & LE (\%) & LC (\%) & $\operatorname{Dg}(\%)$ & Lit. \\
\hline L-PGA-g-Chol & nanoparticle & HSA & N/A & N/A & N/A & 0.065 & [77] \\
\hline \multirow{3}{*}{ PEI-CyD- $g$-Chol } & \multirow{3}{*}{ micelle } & \multirow{3}{*}{ DOX } & \multirow{3}{*}{ N/A } & \multirow{3}{*}{ N/A } & 5.4 & 5.2 & \multirow{3}{*}{ [149] } \\
\hline & & & & & 7.4 & 7.9 & \\
\hline & & & & & 12.8 & 18.6 & \\
\hline Chol-AL-AG & liposome & N/A & 27.0 (N/A) & N/A & N/A & N/A & [150] \\
\hline mPEG-D ${ }_{\text {labile }}$-PAE- $g$-Chol & micelle & DOX & N/A & 53.5 & 11.2 & 55 & [46] \\
\hline Chol-XG & nanogel & PTX & 20000 (N/A) & N/A & N/A & N/A & [151] \\
\hline \multirow{2}{*}{ Dex-Chol } & \multirow{2}{*}{ micelle } & RAPA $10 \%$ & \multirow{2}{*}{43.8 (N/A) } & 79.9 & 7.3 & \multirow{2}{*}{4} & \multirow{2}{*}{ [152] } \\
\hline & & RAPA $20 \%$ & & 90.1 & 12.6 & & \\
\hline mPEG- $b$-P(MBC $\left.78-\left\{g-\mathrm{DMDPTA}_{36} ; g-\mathrm{Chol}_{30}\right\}-c 0-\mathrm{LA}_{110}\right)$ & \multirow{2}{*}{ polyplex } & \multirow{2}{*}{ miRNA-34a } & $43.5(\mathrm{~N} / \mathrm{A})$ & \multirow{2}{*}{ N/A } & \multirow{2}{*}{ N/A } & \multirow{2}{*}{ N/A } & \multirow{2}{*}{ [47] } \\
\hline 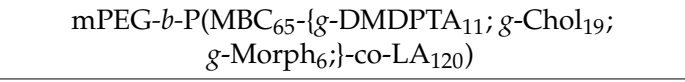 & & & 35.4 (N/A) & & & & \\
\hline \multirow{3}{*}{ Chol-PN } & \multirow{4}{*}{ nanoparticle } & \multirow{4}{*}{ MTX } & \multirow{4}{*}{ N/A } & \multirow{3}{*}{ N/A } & 5.2 & 3.6 & \multirow{4}{*}{ [48] } \\
\hline & & & & & 6.7 & 5.7 & \\
\hline & & & & & 8.6 & 6.7 & \\
\hline mPEG-PLL- $g$-DHPA/Chol-PBA (DHPA:Chol-PBA 1:1) & & & & 55.6 & 13.9 & & \\
\hline Dex-Chol & \multirow{3}{*}{ micelle } & \multirow{3}{*}{ DOX } & \multirow{3}{*}{ N/A } & 41.5 & 6.3 & \multirow{3}{*}{13.0} & \multirow{3}{*}{153} \\
\hline HIS-Dex-Chol (24\% HIS graft ratio) & & & & 46.1 & 7.6 & & \\
\hline HIS-Dex-Chol (46\% HIS graft ratio) & & & & 56.3 & 12.3 & & \\
\hline \multirow{3}{*}{ Chol-g-P(HEMA $10-c o-$ DEAEMA $\left._{25}\right)-b$-PPEGMA 10} & \multirow{6}{*}{ micelle } & $\operatorname{DOX}(12.5 \% w / w)$ & \multirow{3}{*}{$14.3(1.47)$} & 20.0 & 4.1 & \multirow{6}{*}{ N/A } & \multirow{6}{*}{ [49] } \\
\hline & & $\operatorname{DOX}(25 \% w / w)$ & & 38.0 & 8.7 & & \\
\hline & & $\operatorname{DOX}(50 \% w / w)$ & & 30.0 & 13.1 & & \\
\hline & & $\operatorname{DOX}(12.5 \% w / w)$ & & 25.0 & 4.5 & & \\
\hline Chol- $g$-P(HEMA $10-c o-$ DEAEMA $\left._{35}\right)-b$-PPEGMA 10 & & $\operatorname{DOX}(25 \% w / w)$ & $16.4(1.54)$ & 48.5 & 10.8 & & \\
\hline & & $\operatorname{DOX}(50 \% w / w)$ & & 36.7 & 15.5 & & \\
\hline
\end{tabular}


Table 4. Cont

\begin{tabular}{|c|c|c|c|c|c|c|c|}
\hline Polymer & Form of Carrier & Drug or Dye & Mn or Mw (kDa) (Đ) & LE (\%) & LC (\%) & $\operatorname{Dg}(\%)$ & Lit. \\
\hline \multirow{3}{*}{ PAE- $g$-mPEG-Chol } & \multirow{3}{*}{ micelle } & $\operatorname{DOX}(25 \% w / w)$ & \multirow{3}{*}{$8.8(1.90)$} & 25.5 & 9.5 & \multirow{3}{*}{62.0} & \multirow{3}{*}[50]{} \\
\hline & & $\operatorname{DOX}(50 \% w / w)$ & & 60.0 & 28.3 & & \\
\hline & & $\operatorname{DOX}(100 \% w / w)$ & & 52.8 & 30.7 & & \\
\hline HMW-Chol-g-AlgA & \multirow{3}{*}{ nanoparticle } & \multirow{3}{*}{ acetamiprid } & $112.2(\mathrm{~N} / \mathrm{A})$ & 90.8 & \multirow{3}{*}{ N/A } & 4.6 & \multirow{3}{*}{154} \\
\hline MMW-Chol-g-AlgA & & & $64.5(\mathrm{~N} / \mathrm{A})$ & 86.8 & & 5.4 & \\
\hline LMW-Chol-g-AlgA & & & $47.5(\mathrm{~N} / \mathrm{A})$ & 81.0 & & 5.7 & \\
\hline P(NIPAAm-co-NHMAAm)-g-Chol & micelle & Py & $8.1(1.40)$ & $\mathrm{N} / \mathrm{A}$ & 0.4 & $\mathrm{~N} / \mathrm{A}$ & [155] \\
\hline HPC-PEG-Chol-biotin & micelle & PTX & N/A & N/A & 8.4 & 3.6 & [51] \\
\hline CNC-Chol & nanocrystal & FA & $\mathrm{N} / \mathrm{A}$ & 58 & $\mathrm{~N} / \mathrm{A}$ & 17 & [156] \\
\hline Chol-Imi-OS & nanoparticle & CUR & $\mathrm{N} / \mathrm{A}$ & 17.8 & 4.2 & $\mathrm{~N} / \mathrm{A}$ & [157] \\
\hline
\end{tabular}

Abbreviations: acL, acid labile; acS, acid stabile; AG, arabinogalactan; AL, alanine; AlgA, alginic acid; AMPD, 4-(aminomethyl)piperidine; ATRA, all-trans retinoic acid; $\alpha$-azo-caprolactone

$\left(\alpha \mathrm{N}_{3} \mathrm{CL}\right)$; BAC, $N, N$-cystaminebis(acrylamide); CA-Spe, cycloamylose with spermine group; Ce6, chlorin e6; Chol, cholesterol; CholC6, 6-(cholesteryloxycarbonyloxy) hexanol; CholPA, cholestryl 4-pentynoate; CNC, cellulose nanocrystals; coQ10, coenzyme Q10; Cou6, coumarin 6; CS, chitosan; CUR, curcumin; Cyc, cyclam; CyDex, cycloldextrin; DEAEMA, 2-(diethylamino)ethyl methacrylate, Dex, dextrin; Dg, degree of grafting; DHPA, 3-(2;4-dihydroxyphenyl)propionic acid; DMDPTA, $N, N$-dimethyldipropylenetriamine; DOX, doxorubicin; EPO, erythropoietin; F, heptafluorobutyric anhydride; FA, folic acid; FITC-BSA, fluorescein isothiocyanate-labeled bovine serum albumin; GC, glycol chitosan; HA, hyaluronic acid; hbPG, hyperbranched poly(glycerol); HEMA, hydroxyethyl methylacrylate; HIS, histidine; HMW, high molecular weight; HPC, hydroxypropyl cellulose; HSA, human serum albumin; IMC, indomethacine; IMI, imidazole; LA, lactic acid; LMW, low molecular weight; L-PGA, poly(L-glutamic acid); miR-23b, micro RNA-23b; MBC, 5-methyl-5-benzylcarboxyl-1,3-dioxan-2-one; MMW, medium molecular weight; morph, 4-(2-aminoethyl) morpholine; MPC, 2-methacryloyloxyethyl phosphorylcholine; mPEG, (poly(ethylene glycol) methylether methacrylate; MSC, $\mathrm{N}$-methyldiethanolamine-co-diethyl sebacate; MTX, mitoxantrone; NHMAAm, $N$-hydroxylmethylacrylamide; NIPAAm, $N$-isopropylacrylamide; NLS, nuclear localization signal; NPEM, p-nitrophenyloxycarbonylpoly(ethylene glycol)methacrylate; OS, oxidized-starch; PAE, poly( $\beta$-amino ester); PAMD, plerixafor/AMD3100; PBA, poly(3-boronophenyl)carbamate; PDL, w-pentadecanolide; pDNA, plasmid DNA; PEG, poly(ethylene glycol); PEGMA, poly(ethylene glycol) methyl ether methacrylate; PEI, polyethylenimines; PGA, poly(glutamic acid); PHP, w-pentadecanolide; pDNA, plasmid DNA; PEG, poly(ethylene glycol); PEGMA, poly(ethylene glycol) methyl ether methacrylate; PEI, polyethylenimines; PGA, poly(glutamic acid); PHP,
poly[hexamethylene diacrylate- $\beta$-(5-amino-1-pentanol)]; $\gamma$-PGA, poly( $\gamma$-glutamic acid); PLGA, poly(D,L-lactide-co-glycolide); PLL, poly(L-lysine); PMMI, poly(monomethyl itaconate); PN, poly[hexamethylene diacrylate- $\beta$-(5-amino-1-pentanol)]; $\gamma$-PGA, poly( $\gamma$-glutamic acid); PLGA, poly(D,L-lactide-co-glycolide); PLL, poly(L-lysine); PMMI, poly(monomethyl itaconate); PN,
pullulan; PTX, paclitaxel; PURE-G4-OEtOx48, ethoxylated polyurea; PURE-G4-OMeOx48, methoxylated polyurea; PX, piroxicam; Py, pyrene; PyrePA, pyrenylmethyl 4-pentynoate; RAPA, rapamycin; rPAA, bioreducible poly(amidoamine); $\mathrm{SASD}$, supercritical $\mathrm{CO}_{2}$-assisted spray drying; $\mathrm{SDF}$, sildenafil; $\mathrm{SFB}$, sorafenib; SS, disulfide bridge; $\alpha-\mathrm{TOC}$, $\alpha$-tocopherol; $\mathrm{XG}$, xyloglucan. 
The major disadvantage of the post-modification approach is reaction efficiency (usually much below 1) and, as a consequence, a need to use an additional analytical method to determine the degree of post-modification, which increases costs and time of the process. The search for new organic reactions carried out under mild conditions (e.g., Michael addition [144] or orthogonal reactions [158]) and with high yield, the usage of magnetically separable catalysts [159] and the development of new and accurate methods of physicochemical analysis allow us to assume that the post-modification procedure will be further explored.

In the available scientific literature, there are many simplifications, which make it difficult to draw faultless conclusions. The complete physicochemical characterization of the final product is often missing. For instance, the molecular weight and/or dispersity index of the system after post-modification are not determined (Table 4).

\section{Form of Carriers}

There are various forms of drug carriers obtained from polymers bearing cholesteryl moiety/ies (Figure 7).

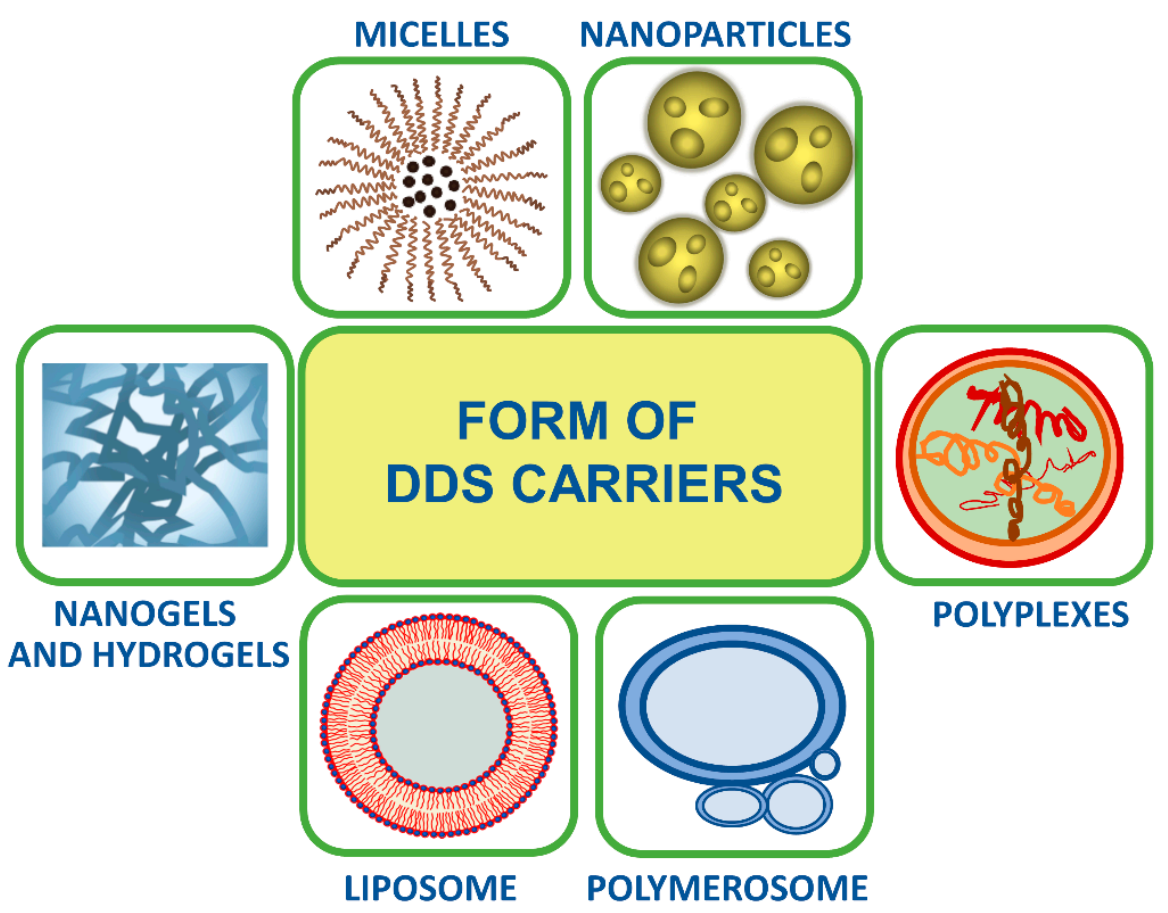

Figure 7. Forms of drug carriers obtained from polymers bearing cholesteryl moiety/ies.

Micellar systems are the simplest constructs in the drug delivery area. They are made of amphiphilic copolymers, the major part of which is hydrophilic. The size of micelles ranges from 5 to $100 \mathrm{~nm}$. These nanospheres are formed in thermodynamic conditions through self-assembly or with an additional factor. They are characterized by a critical micelle concentration (CMC), which is in the range of $10^{-7}$ to $10^{-3} \mathrm{M}$ in water. At the appropriate concentration, the micelles may disintegrate and return to be unimers [160], which may be both the advantage or disadvantage depending on their application. The most common method to obtain micelles used in drug delivery is the precipitation method, where the appropriate selection of the conditions (temperature, concentration, solvent, or their mixture) plays a key role in loading efficiency [161]. During the formation of micelles, it is also possible to encapsulate active substances, which are mostly hydrophobic compounds [162].

Nanoparticles (NPs) are nanostructures made of amphiphilic copolymers with predominated lipophobic part, which are prepared under kinetic conditions. Their sizes are in the range of 50-200 nm. NPs are characterized by higher colloidal stability than micelles, and they do not decay into unimers. 
In drug delivery, they protect against coagulation, aggregation, or phagocytosis, and especially in the case of core/shell type nanoparticles, the core is responsible for the transport of the hydrophobic drug, while the shell acts as a shield and may have a guiding function [160].

Liposomes, i.e., phospholipid vesicles, are spherical structures made of a lipid bilayer. Due to the trapping of water inside the structure, they play the role of transporters of hydrophilic substances in living organisms. Similar structures-polymersomes—can be formed by polymeric amphiphilic compounds, in which the hydrophilic part is in the range of 20-40 wt. \%. As liposomes, they are dedicated to transporting hydrophilic substances. Their sizes vary from 100 to $1000 \mathrm{~nm}$ [163]. One of the methods of producing polymersomes is the film rehydration method [164]. In the literature, there are examples of liposomes composed, inter alia, of cholesterol and other lipids, which are the building block of the double membrane and are not covalently bonded to the polymeric drug carrier. In such a case, the liposome is just a frame or a transporter of the proper working system. Constructing such systems is widespread due to high durability, simplicity of preparation and easy-to-predict behavior [165-169].

Polymer gels are a three-dimensional network of polymer chains, which is formed by chemical or physical cross-linking. A specific group is hydrogels, which are insoluble in water and do not lose their structural integrity, even in the case of high water concentration. Due to their high water absorption, even over $90 \mathrm{wt}$ \%, they are sorbents with great use, for example, in diapers. Hydrogels that occur in the form of nanoparticles are called nanogels. They have diameters of tens to hundreds of nanometers. These are porous materials that can be filled with, for example, drug molecules. It is possible to design properties of hydro and nanogels, such as swelling, degradation, and chemical functionality by the use of various biopolymers or synthetic polymers as well as various cross-linking methods [170-174].

Nanoparticles that are formed by self-assembly of cationic polymers and DNA or RNA are called polyplexes. Such materials are capable of transporting exogenous genetic material into cells in a process called transfection [175].

Cholesterol has many functions in polymeric drug carriers. Its role in cellular uptake is crucial, and cholesterol-containing polymers are characterized by increased cellular uptake in the endocytotic pathway. The mode of action is not clearly described due to the differences in the spatial structure of carriers, encapsulated drugs and pathological targets. Cholesterol stimulates cellular uptake in a lipid rafter-dependent manner [67], by activating the low-density lipoprotein (LDL) receptor [38] and by interacting with glycosphingolipid-rich microdomains in the plasma membrane [127]. Cholesteryl moiety acts as a cell-penetrating agent that stiffens the membrane by embedding into it, which leads to membrane disintegration and tumor growth inhibition [91]. Additionally, the use of cholesterol drug carriers results in higher cellular uptake of the drug. The use of the same dose of loaded drug as the free drug leads to increased apoptosis of neoplastic cells [38,136]. Cholesterol may also function as complexing agent of hydrophobic drugs [76]. Still, there is a gap in the literature on the effect of the number of cholesterol groups in the polymer chain on the stability of plasma membranes and drug delivery.

\section{Drug Encapsulation and Release}

Drug loading capacity (DLC or LC) and drug encapsulation efficiency (DEE or EE) are the basic and most frequently determined parameters in drug delivery and applications. They are expressed as a percentage of the amount of drug-loaded per carrier weight or the amount of drug effectively entrapped in the carrier, respectively. The EE can be calculated as the total weight of the entrapped drug divided by the total weight of the drug added, while the LC is the quotient of the total weight of the entrapped drug and the total weight of the drug-loaded carrier.

$$
\mathrm{EE}(\%)=\frac{\text { total weight of the entrapped drug }}{\text { total weight of drug added }} \times 100
$$




$$
\mathrm{LC}(\%)=\frac{\text { total weight of the entrapped drug }}{\text { total weight of the drug }- \text { loaded carrier }} \times 100
$$

These parameters depend on many factors, including the mass ratio of drug to a vehicle; the method of preparing micelles, nanoparticles, liposomes, and other forms; composition, architecture and arrangement of the polymeric carrier; size and functional groups of the drug molecule; the number of functional groups in the carrier that can complex the drug; the type of drug-carrier interactions; the tendency of polymer chains to twist and self-organize; and the time of dialysis and frequency of water changes.

The variable mass ratio of drug to vehicle is the most frequently studied and easiest to perform the comparison. The same procedure is carried out only by changing the amount of the drug, and the results of such studies show that the research problem is complex. In most cases, as the number of drug increases, EE decreases with increasing LC values $[12,29,57,72,136]$, and, in some cases, both LC and EE values increase [41,152]. It seems logical that by increasing the mass of the added drug, we increase the final loaded mass of the drug in the carrier, but one should consider whether the limit is. The results of the research $[29,69]$ show that there is a critical point at which the maximum value of loading capacity of the DDS is reached, and at some point, there is a drastic decrease in EE from $>95 \%$ to about $70 \%$, while the LC increase is around $1 \%$.

The preparation of nanoparticles has a huge impact on loading capacity and encapsulation efficiency, with solvent selection, temperature, and dialysis time, playing an important role. A solvent, in which both the drug and polymer have the best solubility and can be easily removed without destroying the structures formed, should be selected. A common choice is DMSO or DMF as well as other volatile organic solvents [113]. Dialysis at elevated temperature often leads to an increase in EE [113], while prolonged time reduces both EE and LC values [11].

An effort is being made to determine the relationship between the structure of a carrier and a drug molecule by studies on loading different molecules into the same carrier $[15,19,53]$, and there are also reports on co-loading. It is a very individual matter, and, to date, due to the complexity of the problem, it has not been possible to find a clear answer as to which factors determine the effectiveness of loading. Co loading reduces the LC value $[17,55,71]$, however, it may positively influence EE [17].

Considering the composition of polymeric drug carriers containing a cholesteryl (Chol) moiety, a relevant parameter is the ratio of hydrophilic to hydrophobic parts. The addition of hydrophobic block lowers EE and LC values, whereas hydrophilic units such as PEG [44], folic acid (FA) [136], poly ( $\varepsilon$-caprolactone) (PCL) [58], or histidine (HIS) [153], raise these parameters significantly.

The influence of the cholesterol content in the carrier does not translate unequivocally into the encapsulation efficiency or loading capacity $[48,128,149,154]$. On the other hand, a positive effect is exerted by the change of the carrier's architecture from linear to dendrimer, which raises both the EE and LC values [70].

In vivo release kinetics studies have been described in many articles. Due to the different local environment of neoplastic cells and normal cells, in particular, the slightly acidic environment within neoplastic cells, research is conducted in order to obtain a $\mathrm{pH}$-sensitive carrier that will release drug molecules at a pH below 6 . The conducted research indicates that drug delivery systems not only enable the control of the release depending on $\mathrm{pH}$, but also slow down the release of the active substance over time, which reduces toxicity and reduces the side effects of the used therapies [11,19,20,112,146]. Most of the systems in which cholesterol is covalently bound to the polymer chain by groups are easily hydrolyzed under physiological conditions, and the products of metabolism are an important aspect of the research. Steroid groups ensure the biocompatibility of polymeric carriers and reduce the toxicity of such systems on normal cells [91]. Chytil et al. investigated the amount of cholesterol derivatives released from hydrazone bonding systems. Despite the hydrolysis, the systems were characterized by low toxicity and, at the appropriate $\mathrm{pH}$, a high percentage of drug molecules released [20]. 


\section{Conclusions}

The number of publications that have emerged in recent years shows huge potential hidden in the use of natural products in medical applications. The challenges in the field of drug delivery (specific targeting, intracellular delivery, stimuli-controlled release, etc.) may be met by the application of systems based on polymers containing steroids or their derivatives. Due to the high availability, relatively low price, and hydroxyl group that can easily be derivatized, cholesterol is mainly used for this purpose. The introduction of cholesteryl groups into the structure of the carrier improves its biological properties, biocompatibility, and biodistribution. Polymer chemistry and organic chemistry are developing rapidly, which increases the synthetic possibilities and enables the formation of more complex and more effective systems. Both approaches, polymerization of functional monomers and post-modification, have been successful in the synthesis of polymeric drug carriers containing cholesterol moiety/ies in the main chain or as side chains. Both have advantages and disadvantages that should be taken into account in the course of designing and preparing DDS. Certainly, the molecular weight and molecular weight distribution of the system are factors that have a significant impact on its behavior in the biological environment, and, hence, they should be accurately defined. In this regard, various controlled radical polymerization techniques are the methods of choice as they provide polymers with well-defined properties. In the case of post-modification, the efficiency of the reaction should be taken into account, as it has a huge impact on the properties of the system. The use of diverse polymers and possibility of their modification allows the encapsulation of almost any substance. Polymeric drug carriers containing cholesterol in their structure are mainly tested for the transport of anti-cancer [16,17,48,70-72], anti-fungal [58,152], antibacterial [117], and anti-inflammatory drugs $[44,99,117]$ as well as antioxidants $[15,54,117]$. By using cholesterol-containing systems, endocytosis or fusion of siRNA [100] or pDNA [134] is possible. It is worth noting that there are promising studies on the transmembrane transport of cholesterol-modified siRNA [176].

Author Contributions: Conceptualization, A.Z.W. and P.M.; data collection, D.S., K.H.M. and P.M.; writing original manuscript, A.Z.W., D.S., K.H.M. and P.M.; visualization, A.Z.W. and P.M.; supervision, A.Z.W. All authors have read and agreed to the published version of the manuscript.

Funding: This work was financially supported by the National Science Centre, Poland, grant no. NCN/2016/21/B/ST5/01365.

Conflicts of Interest: All authors declare no conflict of interest.

\section{Abbreviations}

AAA, ascorbyl acrylate; Achol, cholesteryl acrylate; acL, acid labile; acS, acid stabile; ADR, Adriamycin; AECChol, cholesteryl acryloyoxy ethyl carbonate; AEDA, 2-((2-azidoethyl) disulfanyl) ethan-1-amine hydrochloride; AG, arabinogalactan; AL, alanina; AlgA, alginic acid; AmB, amphotericin B; AMPD, 4-(aminomethyl)piperidine; ATRA, all-trans retinoic acid; ATRP, atom transfer radical polymerization; $(\alpha \mathrm{N} 3 \mathrm{CL}), \alpha$-azo-caprolactone; BAC, $N, N$-cystaminebis(acrylamide); BnAAA, benzyl protected ascorbylacrylate; CABA, cabazitaxel; CA-Spe, cycloamylose with spermine group; Ce6, chlorin e6; CF, 5,6-carboxyfluorescein; Chol, cholesterol; CholC6, 6-(cholesteryloxycarbonyloxy) hexanol; CholDEGA, cholesteryl diethyleneglycol acrylate; CholPA, cholestryl 4-pentynoate; CNC, cellulose nanocrystals; coQ10, coenzyme Q10; Cou6, coumarin 6; CPT, S-(+)-camptothecin; CS, chitosan; CUR, curcumin; Cyc, cyclam; CyDex, cycloldextrin; CYS, cystamine; DBU, 1,8-diazabicyklo[5.4.0]undek-7-en, DCC, dicyclohexylcarbodiimide; DEAEMA, 2-(diethylamino)ethyl methacrylate; Dex, dextrin; Dg, degree of grafting; DHPA, 3-(2,4-dihydroxyphenyl)propionic acid; DMA, 1-decyl methacrylate; DMAAm, N,N-dimethylacrylamide; DMAE, 2-(dimethylamino)ethyl 1H-imidazole-1-carboxylate; DMAEMA, 2-(dimethylamino)ethyl methacrylate; DMAP, 4-dimethylaminopyridine, DMDPTA, N,N-dimethyldipropylenetriamine; DMEDA, $N, N$-dimethylaminoethylamine; DOX, doxorubicin; DP7, antimicrobial peptide (VQWRIRVAVIRK); DTXL, docetaxel; DUP1, peptide (CFRPNRAQDYNTN); DUPA, 2-[3-(1,3- dicarboxypropyl) ureido]pentanedioic acid; EDC, 1-(3-dimethylaminopropyl)-3-ethylcarbodiimide; EPO, erythropoietin; F, heptafluorobutyric anhydride; F68, pluronic F68; FA, folic acid; FITC-BSA, fluorescein isothiocyanate-labeled bovine serum albumin; FITC-CM-Dex, fluorescein isothiocyanate carboxymethyl dextran; Fmoc, 9-fluorenylmethoxycarbonyl; GA, glutamic acid; GC, glycyrrhetinic acid; GCS, glycol chitosan; GEM, gemcitabine; HA, hyaluronic acid; hbPG, hyperbranched poly(glycerol); (HE) 5 , histidine-glutamic acid decapeptide; HEMA, hydroxyethyl methylacrylate; HIS, histidine; HMW, high molecular weight; $\mathrm{HPbCD}$, modified 2-hydroxypropyl-b-cyclodextrin macrocycles; HPC, hydroxypropyl cellulose; HPMA, N-(2-hydroxypropyl) methacrylamide; HSA, human serum albumin; Hz, hydrazone; 
IBU, ibuprofen; ICG, indocyanine green; IMC, indomethacine; IMI, imidazole; LA, lactic acid; LC, lecithin; LMW, low molecular weight; L-PGA, poly(L-glutamic acid); Lys, lysine; MAA, methacrylic acid; MAChol, 6-cholesteryloxyhexyl methacrylate; MA- $\varepsilon A h x-C h o l$, cholest-5-en-3 $\beta$-yl 6-methacrylamido hexanohydrazide; MA- $\varepsilon$ Ahx-Chol43, cholest-4-en-3 $\beta$-yl 6-methacrylamido hexanohydrazide; MA- $\varepsilon A h x-C h o l 5 \alpha$, $5 \alpha$-cholestan-3 $\beta$-yl 6-methacrylamido hexanohydrazide; MA- $\varepsilon A h x-L e v C h o l$, cholest-5-en-3 $\beta$-yl-4-oxopentano

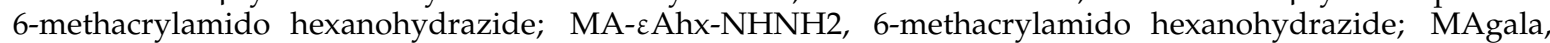
6-Omethacryloyl-D-galactopyranose; MBC, 5-methyl-5-benzylcarboxyl-1,3-dioxan-2-one; MMW, medium molecular weight; morph, 4-(2-aminoethyl) morpholine; MPC, 2-methacryloyloxyethyl phosphorylcholine; mPEG, (poly(ethylene glycol) methylether methacrylate; MSC, N-methyldiethanolamine-co-diethyl sebacate; MTC-Chol, cholesteryl 2-(5-methyl-2-oxo-1;3-dioxane-5-carboxyloyloxy)ethyl carbamate); MTX, mitoxantrone; NAS, N-acryloxysuccinimide; NB, norbornene; NHMAAm, $N$-hydroxylmethylacrylamide; NIPAAm, N-isopropylacrylamide; NLS, nuclear localization signal; NPEM, p-nitrophenyloxycarbonylpoly(ethylene glycol)methacrylate; OC, $\alpha$-tocopherol; OC-ROP, organocatalytic ring-opening polymerization; opB-Chol, cholest-5-en-3 $\beta$-yl-4-(2-oxopropyl)-benzoate; OS, oxidized-starch; PAA, poly(acrylic acid); PAE, poly( $\beta$-amino ester); PAMAM, polyamidoamine; PAMD, plerixafor/AMD3100; PAMPS, poly(2-(acrylamido)-2-methylpropanesulfonic acid) sodium salt; PAsp(DET), poly\{N-[N-(2-aminoethyl)-2-aminoethyl]aspartamide\}; PBA, poly(3-boronophenyl)carbamate; Pbf, 2,2,3,6,7-pentamethyldihydrobenzofuran-5-sulfonyl; PCL, poly( $\varepsilon$-caprolactone); PCLp, polymer-caged lipoplex; PDL, $\omega$-pentadecanolide; PDLA, poly(D-lactide acid); pDNA, plasmid DNA; PEG, poly(ethylene glycol); PEGMA, poly(ethylene glycol) methyl ether methacrylate; PEI, polyethylenimines; PEO, poly(ethylene oxide); PEP, peptide; PF127, Synperonic PE/F 127; PGA, poly $(\gamma$-glutamic acid); PHPMAlac, poly(N-(2-hydroxypropyl)methacrylamide mono/dilactate); PHP, poly[hexamethylene diacrylate- $\beta$-(5-amino-1-pentanol)]; PLA, poly(lactic acid); PLGA, poly(D,L-lactide-co-glycolide); PLGVRK, matrix metalloproteinase-2 responsive peptide; PLL, poly(L-lysine); PLLA, poly(L-lactide acid); PMMI, poly(monomethyl itaconate); PN, pullulan; PplX, protoporphyrin IX; PSO, polyoxyethylene sorbitol oleate; PTX, paclitaxel; PURE-G4-OEtOx48, ethoxylated polyurea; PURE-G4-OMeOx48, methoxylated polyurea; PX, piroxicam; Py, pyrene; PyrePA, pyrenylmethyl 4-pentynoate; QC, quercetin; RAFT, reversible addition-fragmentation chain transfer polymerization; RAPA, rapamycin; RES, resveratrol; $(\mathrm{RG})_{5}$, arginine-glycine decapeptide; RGD, arginylglycylaspartic acid; ROMP, ring-opening metathesis polymerization; $\mathrm{ROP}$, ring-opening polymerization; rPAA, bioreducible poly(amidoamine); SA, succinic anhydride; SASD, supercritical $\mathrm{CO}_{2}$-assisted spray drying; $\mathrm{SDF}$, sildenafil; SFB, sorafenib; SS, disulfide bridge; TMC, trimethylene carbonate; TMX, tamoxifen; $\alpha$-TOC, $\alpha$-tocopherol; TPGS, tocopheryl poly(ethylene glycol) succinate; TPL, triptolide; TPP, triphenylphosphine; uPA, short peptide sequence for urokinase plasminogen activator; XG, xyloglucan.

\section{References}

1. Wilczewska, A.Z.; Niemirowicz, K.; Markiewicz, K.H.; Car, H. Nanoparticles as drug delivery systems. Pharmacol. Rep. 2012, 64, 1020-1037. [CrossRef]

2. Kalepu, S.; Nekkanti, V. Insoluble drug delivery strategies: Review of recent advances and business prospects. Acta Pharm. Sin. B 2015, 5, 442-453. [CrossRef]

3. Jelonek, K.; Kasperczyk, J. Polyesters and polyester carbonates for controlled drug delivery. Part II. Implantable systems. Polimery 2013, 58, 858-863. [CrossRef]

4. Jones, D. Pharmaceutical Applications of Polymers for Drug Delivery; Rapra Review Reports; Rapra Technology: Shrewsbury, UK, 2004; ISBN 978-1-85957-479-9.

5. Nakielski, P. Drug Release Systems Based on Nanofibers; IPPT Reports on Fundamental Technological Research; IPPT PAN: Warsaw, Poland, 2015; ISBN 978-83-89687-93-7.

6. Liu, D.; Yang, F.; Xiong, F.; Gu, N. The Smart Drug Delivery System and Its Clinical Potential. Theranostics 2016, 6, 1306-1323. [CrossRef]

7. El-Sawy, H.S.; Al-Abd, A.M.; Ahmed, T.A.; El-Say, K.M.; Torchilin, V.P. Stimuli-Responsive Nano-Architecture Drug-Delivery Systems to Solid Tumor Micromilieu: Past, Present, and Future Perspectives. ACS Nano 2018, 12, 10636-10664. [CrossRef]

8. Li, B.L.; Li, R.; Zou, H.L.; Ariga, K.; Li, N.B.; Leong, D.T. Engineered functionalized 2D nanoarchitectures for stimuli-responsive drug delivery. Mater. Horiz. 2020, 7, 455-469. [CrossRef]

9. An, H.; Li, M.; Gao, J.; Zhang, Z.; Ma, S.; Chen, Y. Incorporation of biomolecules in Metal-Organic Frameworks for advanced applications. Coord. Chem. Rev. 2019, 384, 90-106. [CrossRef]

10. Bhattacharyya, K.; Mukherjee, S. Fluorescent Metal Nano-Clusters as Next Generation Fluorescent Probes for Cell Imaging and Drug Delivery. Bull. Chem. Soc. Jpn. 2018, 91, 447-454. [CrossRef]

11. Cheng, L.-C.; Jiang, Y.; Xie, Y.; Qiu, L.-L.; Yang, Q.; Lu, H.-Y. Novel amphiphilic folic acid-cholesterol-chitosan micelles for paclitaxel delivery. Oncotarget 2017, 8, 3315-3326. [CrossRef] [PubMed] 
12. Chen, M.; Liu, Y.; Yang, W.; Li, X.; Liu, L.; Zhou, Z.; Wang, Y.; Li, R.; Zhang, Q. Preparation and characterization of self-assembled nanoparticles of 6-O-cholesterol-modified chitosan for drug delivery. Carbohydr. Polym. 2011, 84, 1244-1251. [CrossRef]

13. Tong, X.; Pan, W.; Su, T.; Zhang, M.; Dong, W.; Qi, X. Recent advances in natural polymer-based drug delivery systems. React. Funct. Polym. 2020, 148, 104501. [CrossRef]

14. Nakai, T.; Hirakura, T.; Sakurai, Y.; Shimoboji, T.; Ishigai, M.; Akiyoshi, K. Injectable Hydrogel for Sustained Protein Release by Salt-Induced Association of Hyaluronic Acid Nanogel. Macromol. Biosci. 2012, 12, 475-483. [CrossRef]

15. Huerta-Ángeles, G.; Brandejsová, M.; Novotný, J.; Kopecká, K.; Šógorková, J.; Šmejkalová, D.; Velebný, V. Grafting of steroids to hyaluronan towards the design of delivery systems for antioxidants: The role of hydrophobic core. Carbohydr. Polym. 2018, 193, 383-392. [CrossRef]

16. Zhu, Z.; Li, D.; Li, Y.; Yang, X.; Pan, W. In vitro-in vivo evaluation of hyaluronic acid-based amphiphilic copolymers for tumour targeted delivery: The role of hydrophobic groups. RSC Adv. 2017, 7, 23942-23953. [CrossRef]

17. Zhu, Z.; Li, Y.; Yang, X.; Pan, W.; Pan, H. The reversion of anti-cancer drug antagonism of tamoxifen and docetaxel by the hyaluronic acid-decorated polymeric nanoparticles. Pharmacol. Res. 2017, 126, 84-96. [CrossRef]

18. Fukunaga, K.; Tsutsumi, H.; Mihara, H. Self-Assembling Peptides as Building Blocks of Functional Materials for Biomedical Applications. Bull. Chem. Soc. Jpn. 2019, 92, 391-399. [CrossRef]

19. Jäger, E.; Jäger, A.; Chytil, P.; Etrych, T.; Říhová, B.; Giacomelli, F.C.; Štěpánek, P.; Ulbrich, K. Combination chemotherapy using core-shell nanoparticles through the self-assembly of HPMA-based copolymers and degradable polyester. J. Control. Release 2013, 165, 153-161. [CrossRef]

20. Chytil, P.; Šírová, M.; Kudláčová, J.; Říhová, B.; Ulbrich, K.; Etrych, T. Bloodstream Stability Predetermines the Antitumor Efficacy of Micellar Polymer-Doxorubicin Drug Conjugates with pH-Triggered Drug Release. Mol. Pharm. 2018, 15, 3654-3663. [CrossRef]

21. Filippov, S.K.; Franklin, J.M.; Konarev, P.V.; Chytil, P.; Etrych, T.; Bogomolova, A.; Dyakonova, M.; Papadakis, C.M.; Radulescu, A.; Ulbrich, K.; et al. Hydrolytically Degradable Polymer Micelles for Drug Delivery: A SAXS/SANS Kinetic Study. Biomacromolecules 2013, 14, 4061-4070. [CrossRef]

22. Chytil, P.; Etrych, T.; Kostka, L.; Ulbrich, K. Hydrolytically Degradable Polymer Micelles for Anticancer Drug Delivery to Solid Tumors. Macromol. Chem. Phys. 2012, 213, 858-867. [CrossRef]

23. Zhang, X.; Niebuur, B.-J.; Chytil, P.; Etrych, T.; Filippov, S.K.; Kikhney, A.; Wieland, D.C.F.; Svergun, D.I.; Papadakis, C.M. Macromolecular $p$ HPMA-Based Nanoparticles with Cholesterol for Solid Tumor Targeting: Behavior in HSA Protein Environment. Biomacromolecules 2018, 19, 470-480. [CrossRef]

24. Van Elk, M.; Deckers, R.; Oerlemans, C.; Shi, Y.; Storm, G.; Vermonden, T.; Hennink, W.E. Triggered Release of Doxorubicin from Temperature-Sensitive Poly( $N$-(2-hydroxypropyl)-methacrylamide mono/dilactate) Grafted Liposomes. Biomacromolecules 2014, 15, 1002-1009. [CrossRef]

25. Chiang, Y.-T.; Lo, C.-L. pH-Responsive polymer-liposomes for intracellular drug delivery and tumor extracellular matrix switched-on targeted cancer therapy. Biomaterials 2014, 35, 5414-5424. [CrossRef]

26. Chiang, Y.-T.; Cheng, Y.-T.; Lu, C.-Y.; Yen, Y.-W.; Yu, L.-Y.; Yu, K.-S.; Lyu, S.-Y.; Yang, C.-Y.; Lo, C.-L. Polymer-Liposome Complexes with a Functional Hydrogen-Bond Cross-Linker for Preventing Protein Adsorption and Improving Tumor Accumulation. Chem. Mater. 2013, 25, 4364-4372. [CrossRef]

27. Lou, B.; Connor, K.; Sweeney, K.; Miller, I.S.; O’Farrell, A.; Ruiz-Hernandez, E.; Murray, D.M.; Duffy, G.P.; Wolfe, A.; Mastrobattista, E.; et al. RGD-decorated cholesterol stabilized polyplexes for targeted siRNA delivery to glioblastoma cells. Drug Deliv. Transl. Res. 2019, 9, 679-693. [CrossRef]

28. Kumari, P.; Muddineti, O.S.; Rompicharla, S.V.K.; Ghanta, P.; B B N, A.K.; Ghosh, B.; Biswas, S. Cholesterol-conjugated poly(D,L-lactide)-based micelles as a nanocarrier system for effective delivery of curcumin in cancer therapy. Drug Deliv. 2017, 24, 209-223. [CrossRef]

29. Zhao, Y.; Lin, D.; Wu, F.; Guo, L.; He, G.; Ouyang, L.; Song, X.; Huang, W.; Li, X. Discovery and in Vivo Evaluation of Novel RGD-Modified Lipid-Polymer Hybrid Nanoparticles for Targeted Drug Delivery. Int. J. Mol. Sci. 2014, 15, 17565-17576. [CrossRef]

30. Martin, C.; Marino, N.; Curran, C.; McHale, A.P.; Callan, J.F.; Callan, B. Cholesteryl to improve the cellular uptake of polymersomes within HeLa cells. Int. J. Pharm. 2016, 511, 570-578. [CrossRef] 
31. Venkataraman, S.; Lee, A.L.; Maune, H.T.; Hedrick, J.L.; Prabhu, V.M.; Yang, Y.Y. Formation of Disk- and Stacked-Disk-like Self-Assembled Morphologies from Cholesterol-Functionalized Amphiphilic Polycarbonate Diblock Copolymers. Macromolecules 2013, 46, 4839-4846. [CrossRef]

32. Lee, A.L.Z.; Venkataraman, S.; Sirat, S.B.M.; Gao, S.; Hedrick, J.L.; Yang, Y.Y. The use of cholesterol-containing biodegradable block copolymers to exploit hydrophobic interactions for the delivery of anticancer drugs. Biomaterials 2012, 33, 1921-1928. [CrossRef]

33. Gonzalez-Fajardo, L.; Mahajan, L.H.; Ndaya, D.; Hargrove, D.; Manautou, J.E.; Liang, B.T.; Chen, M.-H.; Kasi, R.M.; Lu, X. Reduced in vivo toxicity of doxorubicin by encapsulation in cholesterol-containing self-assembled nanoparticles. Pharmacol. Res. 2016, 107, 93-101. [CrossRef] [PubMed]

34. Tran, T.-H.; Nguyen, C.T.; Gonzalez-Fajardo, L.; Hargrove, D.; Song, D.; Deshmukh, P.; Mahajan, L.; Ndaya, D.; Lai, L.; Kasi, R.M.; et al. Long Circulating Self-Assembled Nanoparticles from Cholesterol-Containing Brush-Like Block Copolymers for Improved Drug Delivery to Tumors. Biomacromolecules 2014, 15, 4363-4375. [CrossRef] [PubMed]

35. Jia, L.; Cui, D.; Bignon, J.; Di Cicco, A.; Wdzieczak-Bakala, J.; Liu, J.; Li, M.-H. Reduction-Responsive Cholesterol-Based Block Copolymer Vesicles for Drug Delivery. Biomacromolecules 2014, 15, 2206-2217. [CrossRef]

36. Laskar, P.; Samanta, S.; Ghosh, S.K.; Dey, J. In vitro evaluation of pH-sensitive cholesterol-containing stable polymeric micelles for delivery of camptothecin. J. Colloid Interface Sci. 2014, 430, 305-314. [CrossRef]

37. Monajati, M.; Tavakoli, S.; Abolmaali, S.S.; Tamaddon, A. Effect of PEGylation on assembly morphology and cellular uptake of poly ethyleneimine-cholesterol conjugates for delivery of sorafenib tosylate in hepatocellular carcinoma. BioImpacts 2018, 8, 241-252. [CrossRef]

38. Yang, B.; Lv, Y.; Zhu, J.; Han, Y.; Jia, H.; Chen, W.; Feng, J.; Zhang, X.; Zhuo, R. A pH-responsive drug nanovehicle constructed by reversible attachment of cholesterol to PEGylated poly(l-lysine) via catechol-boronic acid ester formation. Acta Biomater. 2014, 10, 3686-3695. [CrossRef]

39. Li, J.; Ma, Y.J.; Wang, Y.; Chen, B.Z.; Guo, X.D.; Zhang, C.Y. Dual redox/pH-responsive hybrid polymer-lipid composites: Synthesis, preparation, characterization and application in drug delivery with enhanced therapeutic efficacy. Chem. Eng. J. 2018, 341, 450-461. [CrossRef]

40. Cheng, W.; Kumar, J.N.; Zhang, Y.; Liu, Y. pH- and Redox-Responsive Poly(ethylene glycol) and Cholesterol-Conjugated Poly(amido amine)s Based Micelles for Controlled Drug Delivery: PH- and Redox-Responsive Poly(amido amine)s Micelles for Controlled Drug Delivery. Macromol. Biosci. 2014, 14, 347-358. [CrossRef]

41. Huang, X.; Liao, W.; Zhang, G.; Kang, S.; Zhang, C.Y. pH-sensitive micelles self-assembled from polymer brush (PAE-g-cholesterol)-b-PEG-b-(PAE-g-cholesterol) for anticancer drug delivery and controlled release. Int. J. Nanomedicine 2017, 12, 2215-2226. [CrossRef]

42. Ghanbarzadeh, S.; Arami, S.; Pourmoazzen, Z.; Khorrami, A. Improvement of the antiproliferative effect of Rapamycin on tumor cell lines by poly(monomethylitaconate)-based $\mathrm{pH}$-sensitive, plasma stable liposomes. Colloids Surf. B Biointerfaces 2014, 115, 323-330. [CrossRef]

43. Ghanbarzadeh, S.; Arami, S.; Pourmoazzen, Z.; Ghasemian-Yadegari, J.; Khorrami, A. Plasma stable, $\mathrm{pH}$-sensitive fusogenic polymer-modified liposomes: A promising carrier for mitoxantrone. J. Biomater. Appl. 2014, 29, 81-92. [CrossRef]

44. Pourmoazzen, Z.; Bagheri, M.; Entezami, A.A.; Koshki, K.N. pH-responsive micelles composed of poly(ethylene glycol) and cholesterol-modified poly(monomethyl itaconate) as a nanocarrier for controlled and targeted release of piroxicam. J. Polym. Res. 2013, 20, 295. [CrossRef]

45. Fritz, T.; Voigt, M.; Worm, M.; Negwer, I.; Müller, S.S.; Kettenbach, K.; Ross, T.L.; Roesch, F.; Koynov, K.; Frey, H.; et al. Orthogonal Click Conjugation to the Liposomal Surface Reveals the Stability of the Lipid Anchorage as Crucial for Targeting. Chem. Eur. J. 2016, 22, 11578-11582. [CrossRef]

46. Xu, M.; Zhang, C.Y.; Wu, J.; Zhou, H.; Bai, R.; Shen, Z.; Deng, F.; Liu, Y.; Liu, J. PEG-Detachable Polymeric Micelles Self-Assembled from Amphiphilic Copolymers for Tumor-Acidity-Triggered Drug Delivery and Controlled Release. ACS Appl. Mater. Interfaces 2019, 11, 5701-5713. [CrossRef]

47. Sharma, S.; Mazumdar, S.; Italiya, K.S.; Date, T.; Mahato, R.I.; Mittal, A.; Chitkara, D. Cholesterol and Morpholine Grafted Cationic Amphiphilic Copolymers for miRNA-34a Delivery. Mol. Pharm. 2018, 15, 2391-2402. [CrossRef] 
48. Yuan, H.; Zhong, W.; Wang, R.; Zhou, P.; Nie, Y.; Hu, W.; Tao, X.; Yang, P. Preparation of Cholesteryl-Modified Aminated Pullulan Nanoparticles to Evaluate Nanoparticle of Hydrophobic Degree on Drug Release and Cytotoxicity. J. Nanomater. 2020, 2020,1-10. [CrossRef]

49. Zhang, C.Y.; Wu, W.S.; Yao, N.; Zhao, B.; Zhang, L.J. pH-sensitive amphiphilic copolymer brush Chol-g-P(HEMA-co-DEAEMA)-b-PPEGMA: Synthesis and self-assembled micelles for controlled anti-cancer drug release. RSC Adv. 2014, 4, 40232-40240. [CrossRef]

50. Zhang, L.; Zhang, C.Y.; Xiong, D.; Sun, Y.; Zhao, B.; Lin, W.J. Self-assembled micelles based on pH-sensitive PAE-g-MPEG-cholesterol block copolymer for anticancer drug delivery. Int. J. Nanomed. 2014, 4923. [CrossRef]

51. Bagheri, M.; Shateri, S.; Niknejad, H.; Entezami, A.A. Thermosensitive biotinylated hydroxypropyl cellulose-based polymer micelles as a nano-carrier for cancer-targeted drug delivery. J. Polym. Res. 2014, 21, 567. [CrossRef]

52. Yang, C.; Liu, S.Q.; Venkataraman, S.; Gao, S.J.; Ke, X.; Chia, X.T.; Hedrick, J.L.; Yang, Y.Y. Structure-directing star-shaped block copolymers: Supramolecular vesicles for the delivery of anticancer drugs. J. Control. Release 2015, 208, 93-105. [CrossRef]

53. Chen, H.-H.; Lu, I.-L.; Liu, T.-I.; Tsai, Y.-C.; Chiang, W.-H.; Lin, S.-C.; Chiu, H.-C. Indocyanine green/doxorubicin-encapsulated functionalized nanoparticles for effective combination therapy against human MDR breast cancer. Colloids Surf. B Biointerfaces 2019, 177, 294-305. [CrossRef]

54. Gao, M.; Yang, Y.; Bergfel, A.; Huang, L.; Zheng, L.; Bowden, T.M. Self-assembly of cholesterol end-capped polymer micelles for controlled drug delivery. J. Nanobiotechnol. 2020, 18. [CrossRef]

55. Andrén, O.C.J.; Zhang, Y.; Lundberg, P.; Hawker, C.J.; Nyström, A.M.; Malkoch, M. Therapeutic Nanocarriers via Cholesterol Directed Self-Assembly of Well-Defined Linear-Dendritic Polymeric Amphiphiles. Chem. Mater. 2017, 29, 3891-3898. [CrossRef]

56. Yu, Y.; He, Y.; Xu, B.; He, Z.; Zhang, Y.; Chen, Y.; Yang, Y.; Xie, Y.; Zheng, Y.; He, G.; et al. Self-Assembled Methoxy Poly(Ethylene Glycol)-Cholesterol Micelles for Hydrophobic Drug Delivery. J. Pharm. Sci. 2013, 102, 1054-1062. [CrossRef]

57. Zeng, S.; Wu, F.; Li, B.; Song, X.; Zheng, Y.; He, G.; Peng, C.; Huang, W. Synthesis, Characterization, and Evaluation of a Novel Amphiphilic Polymer RGD-PEG-Chol for Target Drug Delivery System. Sci. World J. 2014, 2014, 1-10. [CrossRef]

58. Villamil, J.C.; Parra-Giraldo, C.M.; Pérez, L.D. Enhancing the performance of PEG-b-PCL copolymers as precursors of micellar vehicles for amphotericin B through its conjugation with cholesterol. Colloids Surf. Physicochem. Eng. Asp. 2019, 572, 79-87. [CrossRef]

59. Chen, Z.-P.; Xiao, L.; Liu, D.; Feng, M.-S.; Xiao, Y.-Y.; Chen, J.; Li, W.; Li, W.; Cai, B. Synthesis of a novel polymer cholesterol-poly(ethylene glycol) 2000-glycyrrhetinic acid (chol-PEG-GA) and its application in brucine liposome. J. Appl. Polym. Sci. 2011, 124, 4554-4563. [CrossRef]

60. Li, J.; He, Z.; Yu, S.; Li, S.; Ma, Q.; Yu, Y.; Zhang, J.; Li, R.; Zheng, Y.; He, G.; et al. Micelles Based on Methoxy Poly(Ethylene Glycol)Cholesterol Conjugate for Controlled and Targeted Drug Delivery of a Poorly Water Soluble Drug. J. Biomed. Nanotechnol. 2012, 8, 809-817. [CrossRef]

61. Kim, J.H.; Li, Y.; Kim, M.S.; Kang, S.W.; Jeong, J.H.; Lee, D.S. Synthesis and evaluation of biotin-conjugated pH-responsive polymeric micelles as drug carriers. Int. J. Pharm. 2012, 427, 435-442. [CrossRef]

62. Wang, J.; Xu, W.; Ding, J.; Lu, S.; Wang, X.; Wang, C.; Chen, X. Cholesterol-Enhanced Polylactide-Based Stereocomplex Micelle for Effective Delivery of Doxorubicin. Materials 2015, 8, 216-230. [CrossRef] [PubMed]

63. Wang, J.; Xu, W.; Li, S.; Qiu, H.; Li, Z.; Wang, C.; Wang, X.; Ding, J. Polylactide-Cholesterol Stereocomplex Micelle Encapsulating Chemotherapeutic Agent for Improved Antitumor Efficacy and Safety. J. Biomed. Nanotechnol. 2018, 14, 2102-2113. [CrossRef]

64. Cai, L.; Qiu, N.; Li, X.; Luo, K.; Chen, X.; Yang, L.; He, G.; Wei, Y.; Chen, L. A novel truncated basic fibroblast growth factor fragment-conjugated poly (ethylene glycol)-cholesterol amphiphilic polymeric drug delivery system for targeting to the FGFR-overexpressing tumor cells. Int. J. Pharm. 2011, 408, 173-182. [CrossRef] [PubMed]

65. Chen, H.; Wu, F.; Li, J.; Jiang, X.; Cai, L.; Li, X. DUP1 peptide modified micelle efficiently targeted delivery paclitaxel and enhance mitochondrial apoptosis on PSMA-negative prostate cancer cells. SpringerPlus 2016, 5, 362. [CrossRef] 
66. Vabbilisetty, P.; Sun, X.-L. Liposome surface functionalization based on different anchoring lipids via Staudinger ligation. Org. Biomol. Chem. 2014, 12, 1237. [CrossRef]

67. Jia, H.-R.; Zhu, Y.-X.; Xu, K.-F.; Liu, X.; Wu, F.-G. Plasma membrane-anchorable photosensitizing nanomicelles for lipid raft-responsive and light-controllable intracellular drug delivery. J. Control. Release 2018, 286, 103-113. [CrossRef]

68. Oba, M.; Miyata, K.; Osada, K.; Christie, R.J.; Sanjoh, M.; Li, W.; Fukushima, S.; Ishii, T.; Kano, M.R.; Nishiyama, N.; et al. Polyplex micelles prepared from $\omega$-cholesteryl PEG-polycation block copolymers for systemic gene delivery. Biomaterials 2011, 32, 652-663. [CrossRef]

69. Muddineti, O.S.; Vanaparthi, A.; Rompicharla, S.V.K.; Kumari, P.; Ghosh, B.; Biswas, S. Cholesterol and vitamin E-conjugated PEGylated polymeric micelles for efficient delivery and enhanced anticancer activity of curcumin: Evaluation in 2D monolayers and 3D spheroids. Artif. Cells Nanomed. Biotechnol. 2018, 46, 773-786. [CrossRef]

70. Zhang, C.Y.; Chen, Q.; Wu, W.S.; Guo, X.D.; Cai, C.Z.; Zhang, L.J. Synthesis and evaluation of cholesterol-grafted PEGylated peptides with $\mathrm{pH}$-triggered property as novel drug carriers for cancer chemotherapy. Colloids Surf. B Biointerfaces 2016, 142, 55-64. [CrossRef]

71. Zhang, Y.; Xiao, C.; Li, M.; Ding, J.; Yang, C.; Zhuang, X.; Chen, X. Co-delivery of doxorubicin and paclitaxel with linear-dendritic block copolymer for enhanced anti-cancer efficacy. Sci. China Chem. 2014, 57, 624-632. [CrossRef]

72. Barve, A.; Jain, A.; Liu, H.; Zhao, Z.; Cheng, K. Enzyme-responsive polymeric micelles of cabazitaxel for prostate cancer targeted therapy. Acta Biomater. 2020, S1742706120303494. [CrossRef]

73. Chen, D. pH and temperature dual-sensitive liposome gel based on novel cleavable mPEG-Hz-CHEMS polymeric vaginal delivery system. Int. J. Nanomed. 2012, 2621. [CrossRef] [PubMed]

74. Kanamala, M.; Palmer, B.D.; Ghandehari, H.; Wilson, W.R.; Wu, Z. PEG-Benzaldehyde-Hydrazone-Lipid Based PEG-Sheddable pH-Sensitive Liposomes: Abilities for Endosomal Escape and Long Circulation. Pharm. Res. 2018, 35, 154. [CrossRef]

75. Kanamala, M.; Palmer, B.D.; Wilson, W.R.; Wu, Z. Characterization of a smart pH-cleavable PEG polymer towards the development of dual $\mathrm{pH}$-sensitive liposomes. Int. J. Pharm. 2018, 548, 288-296. [CrossRef]

76. Cho, S.-H.; Hong, J.H.; Noh, Y.-W.; Lee, E.; Lee, C.-S.; Lim, Y.T. Raspberry-like poly(gamma;-glutamic acid) hydrogel particles for $\mathrm{pH}$-dependent cell membrane passage and controlled cytosolic delivery of antitumor drugs. Int. J. Nanomedicine 2016, 11, 5621-5632. [CrossRef]

77. Lyu, X.; Zhang, Q.; Liang, D.; Huang, Y. Interaction between human serum albumin and cholesterol-grafted polyglutamate as the potential carriers of protein drugs. Acta Pharm. Sin. B 2019, 9, 186-193. [CrossRef]

78. Terao, K.; Miyake, J.; Watanabe, J.; Ikeda, Y. Regulation of protein loading on poly(trimethylene carbonate), poly(l-lactic acid), and their copolymer: Effect of surface enrichment by polymer crystallinity. Mater. Sci. Eng. C 2012, 32, 988-993. [CrossRef]

79. Wang, Y.; Wang, H.; Liu, G.; Liu, X.; Jin, Q.; Ji, J. Self-Assembly of Near-Monodisperse Redox-Sensitive Micelles from Cholesterol-Conjugated Biomimetic Copolymers: Self-Assembly of Near-Monodisperse Redox-Sensitive Micelles. Macromol. Biosci. 2013, 13, 1084-1091. [CrossRef]

80. Restani, R.B.; Pires, R.F.; Baptista, P.V.; Fernandes, A.R.; Casimiro, T.; Bonifácio, V.D.B.; Aguiar-Ricardo, A. Nano-in-Micro Sildenafil Dry Powder Formulations for the Treatment of Pulmonary Arterial Hypertension Disorders: The Synergic Effect of POxylated Polyurea Dendrimers, PLGA, and Cholesterol. Part. Part. Syst. Charact. 2020, 37, 1900447. [CrossRef]

81. Edlund, U.; Albertsson, A.-C. Degradable Polymer Microspheres for Controlled Drug Delivery. In Degradable Aliphatic Polyesters; Advances in Polymer Science; Springer: Berlin/Heidelberg, Germany, 2002; Volume 157, pp. 67-112. ISBN 978-3-540-42249-5.

82. Zhang, R.; Qin, X.; Kong, F.; Chen, P.; Pan, G. Improving cellular uptake of therapeutic entities through interaction with components of cell membrane. Drug Deliv. 2019, 26, 328-342. [CrossRef]

83. Stewart, M.P.; Langer, R.; Jensen, K.F. Intracellular Delivery by Membrane Disruption: Mechanisms, Strategies, and Concepts. Chem. Rev. 2018, 118, 7409-7531. [CrossRef] [PubMed]

84. Nes, W.D. Biosynthesis of Cholesterol and Other Sterols. Chem. Rev. 2011, 111, 6423-6451. [CrossRef]

85. Sadava, D.E. (Ed.) Life: The Science of Biology, 9th ed.; Sinauer Associates; W. H. Freeman \& Co.: Sunderland, MA, USA; Gordonsville, VA, USA, 2011; ISBN 978-1-4292-1962-4. 
86. Cerqueira, N.M.F.S.A.; Oliveira, E.F.; Gesto, D.S.; Santos-Martins, D.; Moreira, C.; Moorthy, H.N.; Ramos, M.J.; Fernandes, P.A. Cholesterol Biosynthesis: A Mechanistic Overview. Biochemistry 2016, 55, 5483-5506. [CrossRef]

87. Torchilin, V.P. Multifunctional nanocarriers. Adv. Drug Deliv. Rev. 2012, 64, 302-315. [CrossRef]

88. Albuquerque, H.; Santos, C.; Silva, A. Cholesterol-Based Compounds: Recent Advances in Synthesis and Applications. Molecules 2018, 24, 116. [CrossRef] [PubMed]

89. Morzycki, J.W. Recent advances in cholesterol chemistry. Steroids 2014, 83, 62-79. [CrossRef]

90. Zhou, Y.; Briand, V.; Sharma, N.; Ahn, S.; Kasi, R. Polymers Comprising Cholesterol: Synthesis, Self-Assembly, and Applications. Materials 2009, 2, 636-660. [CrossRef]

91. Misiak, P.; Niemirowicz-Laskowska, K.; Markiewicz, K.H.; Misztalewska-Turkowicz, I.; Wielgat, P.; Kurowska, I.; Siemiaszko, G.; Destarac, M.; Car, H.; Wilczewska, A.Z. Evaluation of Cytotoxic Effect of Cholesterol End-Capped Poly(N-Isopropylacrylamide)s on Selected Normal and Neoplastic Cells. Int. J. Nanomedicine 2020, 15, 7263-7278. [CrossRef]

92. Wang, J.-S.; Matyjaszewski, K. Controlled/“living” radical polymerization. atom transfer radical polymerization in the presence of transition-metal complexes. J. Am. Chem. Soc. 1995, 117, 5614-5615. [CrossRef]

93. Kato, M.; Kamigaito, M.; Sawamoto, M.; Higashimura, T. Polymerization of Methyl Methacrylate with the Carbon Tetrachloride/Dichlorotris- (triphenylphosphine)ruthenium(II)/Methylaluminum Bis(2,6-di-tert-butylphenoxide) Initiating System: Possibility of Living Radical Polymerization. Macromolecules 1995, 28, 1721-1723. [CrossRef]

94. Chiefari, J.; Chong, Y.K. (Bill); Ercole, F.; Krstina, J.; Jeffery, J.; Le, T.P.T.; Mayadunne, R.T.A.; Meijs, G.F.; Moad, C.L.; Moad, G.; et al. Living Free-Radical Polymerization by Reversible Addition-Fragmentation Chain Transfer: The RAFT Process. Macromolecules 1998, 31, 5559-5562. [CrossRef]

95. Moad, G. RAFT polymerization to form stimuli-responsive polymers. Polym. Chem. 2017, 8, 177-219. [CrossRef]

96. Messina, M.S.; Messina, K.M.M.; Bhattacharya, A.; Montgomery, H.R.; Maynard, H.D. Preparation of biomolecule-polymer conjugates by grafting-from using ATRP, RAFT, or ROMP. Prog. Polym. Sci. 2020, 100, 101186. [CrossRef]

97. Matyjaszewski, K. Atom Transfer Radical Polymerization (ATRP): Current Status and Future Perspectives. Macromolecules 2012, 45, 4015-4039. [CrossRef]

98. Glaria, A.; Beija, M.; Bordes, R.; Destarac, M.; Marty, J.-D. Understanding the Role of $\omega$-End Groups and Molecular Weight in the Interaction of PNIPAM with Gold Surfaces. Chem. Mater. 2013, 25, 1868-1876. [CrossRef]

99. Zou, T.; Li, F.; Cheng, S.-X.; Zhuo, R.-X. Synthesis and characterization of end-capped biodegradable oligo/poly(trimethylene carbonate)s. J. Biomater. Sci. Polym. Ed. 2006, 17, 1093-1106. [CrossRef]

100. Hong, B.J.; Chipre, A.J.; Nguyen, S.T. Acid-Degradable Polymer-Caged Lipoplex (PCL) Platform for siRNA Delivery: Facile Cellular Triggered Release of siRNA. J. Am. Chem. Soc. 2013, 135, 17655-17658. [CrossRef]

101. Lee, S.-M.; Chen, H.; Dettmer, C.M.; O'Halloran, T.V.; Nguyen, S.T. Polymer-Caged Lipsomes: A pH-Responsive Delivery System with High Stability. J. Am. Chem. Soc. 2007, 129, 15096-15097. [CrossRef]

102. Alves, P.; Hugo, A.A.; Tymczyszyn, E.E.; Ferreira, A.F.; Fausto, R.; Pérez, P.F.; Coelho, J.F.J.; Simões, P.N.; Gómez-Zavaglia, A. Effect of cholesterol-poly(N,N-dimethylaminoethyl methacrylate) on the properties of stimuli-responsive polymer liposome complexes. Colloids Surf. B Biointerfaces 2013, 104, 254-261. [CrossRef]

103. Simões, M.G.; Alves, P.; Carvalheiro, M.; Simões, P.N. Stability effect of cholesterol-poly(acrylic acid) in a stimuli-responsive polymer-liposome complex obtained from soybean lecithin for controlled drug delivery. Colloids Surf. B Biointerfaces 2017, 152, 103-113. [CrossRef]

104. Simões, M.G.; Hugo, A.; Alves, P.; Pérez, P.F.; Gómez-Zavaglia, A.; Simões, P.N. Long term stability and interaction with epithelial cells of freeze-dried $\mathrm{pH}$-responsive liposomes functionalized with cholesterol-poly(acrylic acid). Colloids Surf. B Biointerfaces 2018, 164, 50-57. [CrossRef]

105. Szymanowski, F.; Hugo, A.A.; Alves, P.; Simões, P.N.; Gómez-Zavaglia, A.; Pérez, P.F. Endocytosis and intracellular traffic of cholesterol-PDMAEMA liposome complexes in human epithelial-like cells. Colloids Surf. B Biointerfaces 2017, 156, 38-43. [CrossRef] [PubMed] 
106. Xu, J.-P.; Ji, J.; Chen, W.-D.; Shen, J.-C. Novel biomimetic polymersomes as polymer therapeutics for drug delivery. J. Control. Release 2005, 107, 502-512. [CrossRef]

107. Xu, J.-P.; Ji, J.; Chen, W.-D.; Shen, J.-C. Novel Biomimetic Surfactant: Synthesis and Micellar Characteristics. Macromol. Biosci. 2005, 5, 164-171. [CrossRef]

108. Xu, J.P.; Ji, J.; Chen, W.-D.; Shen, J.C. Biomimetic Amphiphiles for Polymeric Micellar Carrier System. Key Eng. Mater. 2005, 288-289, 465-468. [CrossRef]

109. Zhang, L.; Wang, Q.-R.; Jiang, X.-S.; Cheng, S.-X.; Zhuo, R.-X. Studies on functionalization of poly(E-caprolactone) by a cholesteryl moiety. J. Biomater. Sci. Polym. Ed. 2005, 16, 1095-1108. [CrossRef]

110. Blasco, E.; Sims, M.B.; Goldmann, A.S.; Sumerlin, B.S.; Barner-Kowollik, C. 50th Anniversary Perspective: Polymer Functionalization. Macromolecules 2017, 50, 5215-5252. [CrossRef]

111. Gauthier, M.A.; Gibson, M.I.; Klok, H.-A. Synthesis of Functional Polymers by Post-Polymerization Modification. Angew. Chem. Int. Ed. 2009, 48, 48-58. [CrossRef]

112. Tian, Q.; Shi, J.; Zhao, X.; Di, D.; Deng, Y.; Song, Y. The antitumor efficacy of docetaxel is enhanced by encapsulation in novel amphiphilic polymer cholesterol-coupled tocopheryl polyethylene glycol 1000 succinate micelles. Drug Deliv. Transl. Res. 2017, 7, 642-653. [CrossRef]

113. Varshosaz, J.; Taymouri, S.; Hassanzadeh, F.; Haghjooy Javanmard, S.; Rostami, M. Folated Synperonic-Cholesteryl Hemisuccinate Polymeric Micelles for the Targeted Delivery of Docetaxel in Melanoma. BioMed Res. Int. 2015, 2015, 1-17. [CrossRef]

114. Tian, Y.; Mi, G.; Chen, Q.; Chaurasiya, B.; Li, Y.; Shi, D.; Zhang, Y.; Webster, T.J.; Sun, C.; Shen, Y. Acid-Induced Activated Cell-Penetrating Peptide-Modified Cholesterol-Conjugated Polyoxyethylene Sorbitol Oleate Mixed Micelles for $\mathrm{pH}$-Triggered Drug Release and Efficient Brain Tumor Targeting Based on a Charge Reversal Mechanism. ACS Appl. Mater. Interfaces 2018, 10, 43411-43428. [CrossRef]

115. Deng, Y.; Song, Y.; Tian, Q.; Huang, Z.; Fan, D.; She, Z.; Liu, X.; Cheng, X.; Yu, B. Self-assembled micelles of novel amphiphilic copolymer cholesterol-coupled F68 containing cabazitaxel as a drug delivery system. Int. J. Nanomed. 2014, 2307. [CrossRef]

116. Liu, X.-M.; Yang, Y.-Y.; Leong, K.W. Thermally responsive polymeric micellar nanoparticles self-assembled from cholesteryl end-capped random poly(N-isopropylacrylamide-co- $\mathrm{N}, \mathrm{N}$-dimethylacrylamide): Synthesis, temperature-sensitivity, and morphologies. J. Colloid Interface Sci. 2003, 266, 295-303. [CrossRef]

117. Angarita, A.V.; Umaña-Perez, A.; Perez, L.D. Enhancing the performance of PEG- $b$-PCL-based nanocarriers for curcumin through its conjugation with lipophilic biomolecules. J. Bioact. Compat. Polym. 2020, 35, 399-413. [CrossRef]

118. Badwaik, V.D.; Aicart, E.; Mondjinou, Y.A.; Johnson, M.A.; Bowman, V.D.; Thompson, D.H. Structure-property relationship for in vitro siRNA delivery performance of cationic 2-hydroxypropyl- $\beta$-cyclodextrin: PEG-PPG-PEG polyrotaxane vectors. Biomaterials 2016, 84, 86-98. [CrossRef]

119. Kim, G.; Piao, C.; Oh, J.; Lee, M. Self-assembled polymeric micelles for combined delivery of anti-inflammatory gene and drug to the lungs by inhalation. Nanoscale 2018, 10, 8503-8514. [CrossRef]

120. Lin, S.-Y.; Zhao, W.-Y.; Tsai, H.-C.; Hsu, W.-H.; Lo, C.-L.; Hsiue, G.-H. Sterically Polymer-Based Liposomal Complexes with Dual-Shell Structure for Enhancing the siRNA Delivery. Biomacromolecules 2012, 13, 664-675. [CrossRef]

121. Basel, M.T.; Shrestha, T.B.; Troyer, D.L.; Bossmann, S.H. Protease-Sensitive, Polymer-Caged Liposomes: A Method for Making Highly Targeted Liposomes Using Triggered Release. ACS Nano 2011, 5, 2162-2175. [CrossRef]

122. Zhang, R.; Tang, L.; Tian, Y.; Ji, X.; Hu, Q.; Zhou, B.; Zhenyu, D.; Heng, X.; Yang, L. Cholesterol-modified DP7 enhances the effect of individualized cancer immunotherapy based on neoantigens. Biomaterials 2020, 241, 119852. [CrossRef]

123. Liu, Y.; Wang, Y.; Zhuang, D.; Yang, J.; Yang, J. Bionanoparticles of amphiphilic copolymers polyacrylate bearing cholesterol and ascorbate for drug delivery. J. Colloid Interface Sci. 2012, 377, 197-206. [CrossRef] [PubMed]

124. Lynge, M.E.; Baekgaard Laursen, M.; Hosta-Rigau, L.; Jensen, B.E.B.; Ogaki, R.; Smith, A.A.A.; Zelikin, A.N.; Städler, B. Liposomes as Drug Deposits in Multilayered Polymer Films. ACS Appl. Mater. Interfaces 2013, 5, 2967-2975. [CrossRef] [PubMed]

125. Zhang, Y.; Lynge, M.E.; Nielsen, M.B.; Schattling, P.S.; Han, X.; Städler, B. Patterned Liposome-Polymer Composite Coatings. ChemNanoMat 2016, 2, 822-829. [CrossRef] 
126. Sevimli, S.; Inci, F.; Zareie, H.M.; Bulmus, V. Well-Defined Cholesterol Polymers with pH-Controlled Membrane Switching Activity. Biomacromolecules 2012, 13, 3064-3075. [CrossRef]

127. Sevimli, S.; Sagnella, S.; Macmillan, A.; Whan, R.; Kavallaris, M.; Bulmus, V.; Davis, T.P. The endocytic pathway and therapeutic efficiency of doxorubicin conjugated cholesterol-derived polymers. Biomater. Sci. 2015, 3, 323-335. [CrossRef] [PubMed]

128. Wang, Z.; Luo, T.; Sheng, R.; Li, H.; Sun, J.; Cao, A. Amphiphilic Diblock Terpolymer PMAgala- $b$-P(MAA-co-MAChol)s with Attached Galactose and Cholesterol Grafts and Their Intracellular pH-Responsive Doxorubicin Delivery. Biomacromolecules 2016, 17, 98-110. [CrossRef] [PubMed]

129. Dong, M.; Chen, J.; Yang, J.; Jiang, W.; Han, H.; Li, Q.; Yang, Y. Chemoenzymatic synthesis of a cholesterol-g-poly(amine-co-ester) carrier for p53 gene delivery to inhibit the proliferation and migration of tumor cells. New J. Chem. 2018, 42, 13541-13548. [CrossRef]

130. Chen, J.; Jiang, W.; Han, H.; Yang, J.; Chen, W.; Wang, Y.; Tang, J.; Li, Q. Chemoenzymatic Synthesis of Cholesterol-g-Poly(amine-co-ester) Amphiphilic Copolymer as a Carrier for miR-23b Delivery. ACS Macro Lett. 2017, 6, 523-528. [CrossRef]

131. Liu, Y.; Chen, D.; Li, J.; Xia, D.; Yu, M.; Tao, J.; Zhang, X.; Li, L.; Gan, Y. NPC1L1-Targeted Cholesterol-Grafted Poly( $\beta$-Amino Ester)/pDNA Complexes for Oral Gene Delivery. Adv. Healthc. Mater. 2019, 8, 1800934. [CrossRef]

132. Valencia-Serna, J.; Kucharski, C.; Chen, M.; Kc, R.; Jiang, X.; Brandwein, J.; Uludağ, H. siRNA-mediated BCR-ABL silencing in primary chronic myeloid leukemia cells using lipopolymers. J. Control. Release 2019, 310, 141-154. [CrossRef]

133. Lin, Y.-K.; Fang, J.-Y.; Wang, S.-W.; Lee, R.-S. Synthesis and characterization of triple-responsive PNiPAAm-S-S-P( $\alpha$ N3CL-g-alkyne $)$ copolymers bearing cholesterol and fluorescence monitor. React. Funct. Polym. 2018, 130, 29-42. [CrossRef]

134. Chen, J.; Yu, Z.; Chen, H.; Gao, J.; Liang, W. Transfection efficiency and intracellular fate of polycation liposomes combined with protamine. Biomaterials 2011, 32, 1412-1418. [CrossRef]

135. Ding, K.; Li, R.; Ma, Y.; Li, N.; Zhang, T.; Cheng-Mei, X.; Jiang, H.-T.; Gong, Y.-K. Folate Ligand Orientation Optimized during Cell Membrane Mimetic Micelle Formation for Enhanced Tumor Cell Targeting. Langmuir 2019, 35, 1257-1265. [CrossRef]

136. Jiang, H.-T.; Ding, K.; Meng, F.-N.; Bao, L.-L.; Chai, Y.-D.; Gong, Y.-K. Anti-phagocytosis and tumor cell targeting micelles prepared from multifunctional cell membrane mimetic polymers. J. Mater. Chem. B 2016, 4, 5464-5474. [CrossRef]

137. Remant, K.; Thapa, B.; Valencia-Serna, J.; Domun, S.S.; Dimitroff, C.; Jiang, X.; Uludağ, H. Cholesterol grafted cationic lipopolymers: Potential siRNA carriers for selective chronic myeloid leukemia therapy. J. Biomed. Mater. Res. A 2020, 108, 565-580. [CrossRef]

138. Kc, R.; Thapa, B.; Ubeda, A.; Jiang, X.; Uludağ, H. BCR-Abl Silencing by siRNA: A Potent Approach to Sensitize Chronic Myeloid Leukemia Cells to Tyrosine Kinase Inhibitor Therapy. Stem Cells Dev. 2019, 28, 734-744. [CrossRef] [PubMed]

139. Fujii, H.; Shin-Ya, M.; Takeda, S.; Hashimoto, Y.; Mukai, S.; Sawada, S.; Adachi, T.; Akiyoshi, K.; Miki, T.; Mazda, O. Cycloamylose-nanogel drug delivery system-mediated intratumor silencing of the vascular endothelial growth factor regulates neovascularization in tumor microenvironment. Cancer Sci. 2014, 105, 1616-1625. [CrossRef] [PubMed]

140. Wu, P.; Luo, X.; Wu, H.; Yu, F.; Wang, K.; Sun, M.; Oupicky, D. Cholesterol Modification Enhances Antimetastatic Activity and siRNA Delivery Efficacy of Poly(ethylenimine)-Based CXCR4 Antagonists. Macromol. Biosci. 2018, 18, 1800234. [CrossRef]

141. Sun, Y.-D.; Zhu, Y.-X.; Zhang, X.; Jia, H.-R.; Xia, Y.; Wu, F.-G. Role of Cholesterol Conjugation in the Antibacterial Photodynamic Therapy of Branched Polyethylenimine-Containing Nanoagents. Langmuir 2019, 35, 14324-14331. [CrossRef] [PubMed]

142. Yu, J.; Xie, X.; Xu, X.; Zhang, L.; Zhou, X.; Yu, H.; Wu, P.; Wang, T.; Che, X.; Hu, Z. Development of dual ligand-targeted polymeric micelles as drug carriers for cancer therapy in vitro and in vivo. J. Mater. Chem. B 2014, 2, 2114. [CrossRef] [PubMed]

143. Wu, P.; Luo, X.; Wu, H.; Zhang, Q.; Wang, K.; Sun, M.; Oupicky, D. Combined Hydrophobization of Polyethylenimine with Cholesterol and Perfluorobutyrate Improves siRNA Delivery. Bioconjug. Chem. 2020, 31, 698-707. [CrossRef] [PubMed] 
144. Wang, Y.; Li, J.; Chen, Y.; Oupický, D. Balancing polymer hydrophobicity for ligand presentation and siRNA delivery in dual function CXCR4 inhibiting polyplexes. Biomater. Sci. 2015, 3, 1114-1123. [CrossRef] [PubMed]

145. Morimoto, N.; Hirano, S.; Takahashi, H.; Loethen, S.; Thompson, D.H.; Akiyoshi, K. Self-Assembled pH-Sensitive Cholesteryl Pullulan Nanogel As a Protein Delivery Vehicle. Biomacromolecules 2013, 14, 56-63. [CrossRef] [PubMed]

146. Liu, J.; Li, J.; Liu, T. Fabrication of Mixed Polymeric Micelles Based on Stimuli-Responsive Amphiphilic Copolymers for Drug Delivery and Controlled Release. Nano 2020, 15, 2050040. [CrossRef]

147. Chen, C.-J.; Wang, J.-C.; Zhao, E.-Y.; Gao, L.-Y.; Feng, Q.; Liu, X.-Y.; Zhao, Z.-X.; Ma, X.-F.; Hou, W.-J.; Zhang, L.-R.; et al. Self-assembly cationic nanoparticles based on cholesterol-grafted bioreducible poly(amidoamine) for siRNA delivery. Biomaterials 2013, 34, 5303-5316. [CrossRef]

148. Gao, L.-Y.; Liu, X.-Y.; Chen, C.-J.; Wang, J.-C.; Feng, Q.; Yu, M.-Z.; Ma, X.-F.; Pei, X.-W.; Niu, Y.-J.; Qiu, C.; et al. Core-Shell type lipid/rPAA-Chol polymer hybrid nanoparticles for in vivo siRNA delivery. Biomaterials 2014, 35, 2066-2078. [CrossRef]

149. Shen, J.; Wang, Q.; Hu, Q.; Li, Y.; Tang, G.; Chu, P.K. Restoration of chemosensitivity by multifunctional micelles mediated by P-gp siRNA to reverse MDR. Biomaterials 2014, 35, 8621-8634. [CrossRef] [PubMed]

150. Pathak, P.O.; Nagarsenker, M.S.; Barhate, C.R.; Padhye, S.G.; Dhawan, V.V.; Bhattacharyya, D.; Viswanathan, C.L.; Steiniger, F.; Fahr, A. Cholesterol anchored arabinogalactan for asialoglycoprotein receptor targeting: Synthesis, characterization, and proof of concept of hepatospecific delivery. Carbohydr. Res. 2015, 408, 33-43. [CrossRef]

151. Sawada, S.; Yukawa, H.; Takeda, S.; Sasaki, Y.; Akiyoshi, K. Self-assembled nanogel of cholesterol-bearing xyloglucan as a drug delivery nanocarrier. J. Biomater. Sci. Polym. Ed. 2017, 28, 1183-1198. [CrossRef]

152. Shaki, H.; Ganji, F.; Kempen, P.J.; Dolatshahi-Pirouz, A.; Vasheghani-Farahani, E. Self-assembled amphiphilic-dextran nanomicelles for delivery of rapamycin. J. Drug Deliv. Sci. Technol. 2018, 44, 333-341. [CrossRef]

153. Yao, X.; Chen, L.; Chen, X.; He, C.; Zheng, H.; Chen, X. Intercellular pH-responsive histidine modified dextran-g-cholesterol micelle for anticancer drug delivery. Colloids Surf. B Biointerfaces 2014, 121, $36-43$. [CrossRef]

154. Zhao, X.; Li, J.; Feng, Y.; Yu, G.; Zhou, Q.; He, F.; Xiao, D.; Chen, K.; Zhang, L. Self-aggregation behavior of hydrophobic sodium alginate derivatives in aqueous solution and their application in the nanoencapsulation of acetamiprid. Int. J. Biol. Macromol. 2018, 106, 418-424. [CrossRef]

155. Liu, X.-M.; Pramoda, K.P.; Yang, Y.-Y.; Chow, S.Y.; He, C. Cholesteryl-grafted functional amphiphilic poly(N-isopropylacrylamide-co-N-hydroxylmethylacrylamide): Synthesis, temperature-sensitivity, self-assembly and encapsulation of a hydrophobic agent. Biomaterials 2004, 25, 2619-2628. [CrossRef]

156. Pourmoazzen, Z.; Sadeghifar, H.; Chen, J.; Yang, G.; Zhang, K.; Lucia, L. The morphology, self-assembly, and host-guest properties of cellulose nanocrystals surface grafted with cholesterol. Carbohydr. Polym. 2020, 233, 115840. [CrossRef]

157. Xu, Y.; Zi, Y.; Lei, J.; Mo, X.; Shao, Z.; Wu, Y.; Tian, Y.; Li, D.; Mu, C. pH-Responsive nanoparticles based on cholesterol/imidazole modified oxidized-starch for targeted anticancer drug delivery. Carbohydr. Polym. 2020, 233, 115858. [CrossRef]

158. Devaraj, N.K. The Future of Bioorthogonal Chemistry. ACS Cent. Sci. 2018, 4, 952-959. [CrossRef]

159. Misztalewska-Turkowicz, I.; Markiewicz, K.H.; Michalak, M.; Wilczewska, A.Z. NHC-copper complexes immobilized on magnetic nanoparticles: Synthesis and catalytic activity in the CuAAC reactions. J. Catal. 2018, 362, 46-54. [CrossRef]

160. Cho, H.K.; Cheong, I.W.; Lee, J.M.; Kim, J.H. Polymeric nanoparticles, micelles and polymersomes from amphiphilic block copolymer. Korean J. Chem. Eng. 2010, 27, 731-740. [CrossRef]

161. Liu, Y.; Yang, G.; Baby, T.; Chen, D.; Weitz, D.A.; Zhao, C. Stable Polymer Nanoparticles with Exceptionally High Drug Loading by Sequential Nanoprecipitation. Angew. Chem. Int. Ed. 2020, 59, 4720-4728. [CrossRef]

162. Allen, C.; Maysinger, D.; Eisenberg, A. Nano-engineering block copolymer aggregates for drug delivery. Colloids Surf. B Biointerfaces 1999, 16, 3-27. [CrossRef]

163. Mabrouk, E.; Cuvelier, D.; Pontani, L.-L.; Xu, B.; Lévy, D.; Keller, P.; Brochard-Wyart, F.; Nassoy, P.; Li, M.-H. Formation and material properties of giant liquid crystal polymersomes. Soft Matter 2009, 5, 1870. [CrossRef] 
164. Letchford, K.; Burt, H. A review of the formation and classification of amphiphilic block copolymer nanoparticulate structures: Micelles, nanospheres, nanocapsules and polymersomes. Eur. J. Pharm. Biopharm. 2007, 65, 259-269. [CrossRef]

165. Yu, J.; Wang, Y.; Zhou, S.; Li, J.; Wang, J.; Chi, D.; Wang, X.; Lin, G.; He, Z.; Wang, Y. Remote loading paclitaxel-doxorubicin prodrug into liposomes for cancer combination therapy. Acta Pharm. Sin. B 2020, 10, 1730-1740. [CrossRef]

166. Liu, A.; Wang, H.; Hou, X.; Ma, Y.; Yang, G.; Hou, Y.; Ding, Y. Combinatory antitumor therapy by cascade targeting of a single drug. Acta Pharm. Sin. B 2020, 10, 667-679. [CrossRef] [PubMed]

167. Scopel, R.; Falcão, M.A.; Cappellari, A.R.; Morrone, F.B.; Guterres, S.S.; Cassel, E.; Kasko, A.M.; Vargas, R.M.F. Lipid-polymer hybrid nanoparticles as a targeted drug delivery system for melanoma treatment. Int. J. Polym. Mater. Polym. Biomater. 2020, 1-12. [CrossRef]

168. Krstić, M.; Manić, L.; Martić, N.; Vasiljević, D.; Mračević, S.Đ.; Vukmirović, S.; Rašković, A. Binary polymeric amorphous carvedilol solid dispersions: In vitro and in vivo characterization. Eur. J. Pharm. Sci. 2020, 150, 105343. [CrossRef] [PubMed]

169. Han, Z.; Lv, W.; Li, Y.; Chang, J.; Zhang, W.; Liu, C.; Sun, J. Improving Tumor Targeting of Exosomal Membrane-Coated Polymeric Nanoparticles by Conjugation with Aptamers. ACS Appl. Biol. Mater. 2020, 3, 2666-2673. [CrossRef]

170. Bencherif, S.A.; Siegwart, D.J.; Srinivasan, A.; Horkay, F.; Hollinger, J.O.; Washburn, N.R.; Matyjaszewski, K. Nanostructured hybrid hydrogels prepared by a combination of atom transfer radical polymerization and free radical polymerization. Biomaterials 2009, 30, 5270-5278. [CrossRef]

171. Kabanov, A.V.; Vinogradov, S.V. Nanogels as Pharmaceutical Carriers: Finite Networks of Infinite Capabilities. Angew. Chem. Int. Ed. 2009, 48, 5418-5429. [CrossRef]

172. Lee, H.; Mok, H.; Lee, S.; Oh, Y.-K.; Park, T.G. Target-specific intracellular delivery of siRNA using degradable hyaluronic acid nanogels. J. Control. Release 2007, 119, 245-252. [CrossRef]

173. Hayashi, H.; Iijima, M.; Kataoka, K.; Nagasaki, Y. pH-Sensitive Nanogel Possessing Reactive PEG Tethered Chains on the Surface. Macromolecules 2004, 37, 5389-5396. [CrossRef]

174. Warren, D.S.; Sutherland, S.P.H.; Kao, J.Y.; Weal, G.R.; Mackay, S.M. The Preparation and Simple Analysis of a Clay Nanoparticle Composite Hydrogel. J. Chem. Educ. 2017, 94, 1772-1779. [CrossRef]

175. D'Andrea, C.; Pezzoli, D.; Malloggi, C.; Candeo, A.; Capelli, G.; Bassi, A.; Volonterio, A.; Taroni, P.; Candiani, G. The study of polyplex formation and stability by time-resolved fluorescence spectroscopy of SYBR Green I-stained DNA. Photochem. Photobiol. Sci. 2014, 13, 1680-1689. [CrossRef]

176. Zheng, Y.; Tai, W. Insight into the siRNA transmembrane delivery-From cholesterol conjugating to tagging. WIREs Nanomed. Nanobiotechnol. 2020, 12. [CrossRef]

Publisher's Note: MDPI stays neutral with regard to jurisdictional claims in published maps and institutional affiliations.

(C) 2020 by the authors. Licensee MDPI, Basel, Switzerland. This article is an open access article distributed under the terms and conditions of the Creative Commons Attribution (CC BY) license (http://creativecommons.org/licenses/by/4.0/). 